\title{
Monocarboxylate transporter 1 in Schwann cells contributes to maintenance of sensory nerve myelination during aging
}

\author{
Mithilesh Kumar Jha ${ }^{1}$, Youngjin Lee ${ }^{1,2}$, Katelyn A. Russell ${ }^{1}$, Fang Yang ${ }^{1}$, Raha M. \\ Dastgheyb ${ }^{1}$, Pragney Deme ${ }^{1}$, Xanthe H. Ament ${ }^{1}$, Weiran Chen ${ }^{1}$, Ying Liu ${ }^{1}$, Yun Guan ${ }^{3,4}$, \\ Michael J. Polydefkis ${ }^{1}$, Ahmet Hoke ${ }^{1}$, Norman J. Haughey ${ }^{1}$, Jeffrey D. Rothstein ${ }^{1,5}$, Brett M. \\ Morrison $^{1}$
}

${ }^{1}$ Department of Neurology, Johns Hopkins University School of Medicine, Baltimore, Maryland ${ }^{2}$ Department of Biomedical Sciences, City University of Hong Kong, Hong Kong SAR, Hong Kong ${ }^{3}$ Department of Anesthesiology and Critical Care Medicine, Johns Hopkins University School of Medicine, Baltimore, Maryland ${ }^{4}$ Department of Neurological Surgery, Johns Hopkins University School of Medicine, Baltimore, Maryland ${ }^{5}$ Brain Science Institute, Johns Hopkins University, Baltimore, Maryland

\begin{abstract}
Schwann cell (SC)-specific monocarboxylate transporter 1 (MCT1) knockout mice were generated by mating MCT1 $1^{f / f}$ mice with myelin protein zero $(\mathrm{P} 0)$-Cre mice. $\mathrm{P} 0-\mathrm{Cre}^{+/-}, \mathrm{MCT} 1^{f / f}$ mice have no detectable early developmental defects, but develop hypomyelination and reduced conduction velocity in sensory, but not motor, peripheral nerves during maturation and aging. Furthermore, reduced mechanical sensitivity is evident in aged $\mathrm{P} 0-\mathrm{Cre}^{+/-}, \mathrm{MCT} 1 / f /$ mice. MCT1 deletion in SCs impairs both their glycolytic and mitochondrial functions, leading to altered lipid metabolism of triacylglycerides, diacylglycerides, and sphingomyelin, decreased expression of myelin-associated glycoprotein, and increased expression of c-Jun and p75-neurotrophin receptor, suggesting a regression of SCs to a less mature developmental state. Taken together, our results define the contribution of SC MCT1 to both SC metabolism and peripheral nerve maturation and aging.
\end{abstract}

\section{Keywords}

lactate; MCT1; metabolism; monocarboxylate transporter; myelination; peripheral nerve; Schwann cell; sensory axons; triacylglycerides

Correspondence: Brett M. Morrison, Department of Neurology, Johns Hopkins University School of Medicine, 855 North Wolfe Street, Rangos 248, Baltimore, MD 21205. bmorris7@jhmi.edu.

AUTHOR CONTRIBUTIONS

M.K.J. conceived and performed experiments, analyzed data, and wrote the manuscript. Y.L., K.A.R., F.Y., R.M.D., P.D., X.H.A., W.C., and Y.L. performed some of the experiments and/or data analysis. Y.G., M.J.P., A.H., N.J.H., and J.D.R. assisted with resources. Y.G., A.H., N.J.H., and J.D.R. provided expertise and feedback. B.M.M. secured funding, conceived and supervised the study, analyzed data, and wrote the manuscript.

CONFLICT OF INTEREST

The authors declare no competing financial interests.

SUPPORTING INFORMATION

Additional supporting information may be found online in the Supporting Information section at the end of this article. 


\section{1 | INTRODUCTION}

Structural maintenance and proper functioning of the peripheral nervous system (PNS) require high metabolic supply. Aging peripheral nerves have progressive reduction in energy stores (Low, Schmelzer, \& Ward, 1986) as well as blood flow due to reduction of microvasculature caliber (Kihara, Nickander, \& Low, 1991). Although Schwann cells (SCs) play a critical role in myelinating axons of peripheral nerves and facilitating saltatory conduction, growing evidence suggests that similar to oligodendrocytes and astrocytes in the central nervous system (CNS; Funfschilling et al., 2012; Lee et al., 2012; Pellerin \& Magistretti, 1994), SCs may also be a primary metabolic supporter to axons in the PNS (Beirowski et al., 2014; Vega, Martiel, Drouhault, Burckhart, \& Coles, 2003). Such metabolic support is quite important for maintenance of axonal integrity and function in the PNS, where there are long axons that have distal regions relatively isolated from their cell bodies. Emerging evidence outlines the critical role of the axon-SC relationship and associated bioenergetic crosstalk in peripheral nerve health and diseases. Transfer of metabolic substrates from SCs to axons has been previously reported in rodent models (Vega et al., 2003), and SC metabolism has been found to be critical for developmental myelination, myelin maintenance, and support of axon integrity and function (Beirowski et al., 2014; Viader et al., 2011; Yin et al., 2016).

Uninterrupted energy supply is necessary for the survival of peripheral axons. The presence of glycogen, which is the primary intracellular metabolic storage molecule, within SCs establishes this cell type as an important source of energy substrates, primarily in the form of lactate, for myelinated axons during aglycemia (Brown, Evans, Black, \& Ransom, 2012). Growing evidence suggests a critical role of lactate as a dynamic metabolite in health and diseases of peripheral nerves (Beirowski et al., 2014; Jha \& Morrison, 2018). During catabolic processes, glycogen present in SCs is metabolized into lactate that may be transported to axons via monocarboxylate transporters (MCTs), which are bi-directional transporters of protons and monocarboxylates, including lactate, across plasma membranes. Within axons, lactate or other monocarboxylates may then be oxidized to pyruvate and metabolized through the Krebs cycle for ATP production (Beirowski et al., 2014; Brown et al., 2012). Monocarboxylate transporter 1 (MCT1) is the most abundantly expressed lactate transporter in peripheral nerves, being expressed in SCs along with other cell types (Domenech-Estevez et al., 2015; Morrison et al., 2015; Takebe, Nio-Kobayashi, TakahashiIwanaga, \& Iwanaga, 2008). We have previously reported that MCT1 is critical for nerve regeneration following injury, and this altered regeneration is potentially due to impairment of intrinsic SC biology necessary for proliferation, de-differentiation, or cytokine release (Morrison et al., 2015). Alternatively, reduced nerve regeneration may be due to altered metabolic support from SCs to regenerating axons. Though not yet specifically investigated in SCs in vivo, oligodendrocyte progenitor cells (OPCs) in the CNS utilize lactate through MCTs to promote proliferation and differentiation (Ichihara et al., 2017). Surprisingly, downregulation of MCT1 in SCs in vitro by lentiviral shRNAs reportedly increases myelination in DRG neuron/SCs myelinating co-cultures (Domenech-Estevez et al., 2015). Hence, the exact role of MCT1 expressed in SCs in peripheral nerve biology is not yet clear. 
To directly interrogate the role of MCT1 in SCs in vivo, we have developed P0-Cre ${ }^{+/}$, $\mathrm{MCT}^{\mathrm{f} / \mathrm{f}}$ mice (P0-cMCT1 KO), which have SC-specific ablation of MCT1, by mating newly generated $\mathrm{MCT} 1^{f / f}$ mice with myelin protein zero Cre-recombinase (P0-Cre) mice. In this study, we evaluate the integrity of peripheral nerve axons and myelin, function of peripheral nerves by both electrophysiologic measurements and behavior, and characterize the specific metabolic alterations in $\mathrm{P} 0$-cMCT1 $\mathrm{KO}$ mice during development and aging. We find that SC-specific MCT1 deletion impairs glycolytic and mitochondrial functions, depletes the nerve of critical lipids, and results in hypomyelination and functional deficits in sensory, but not motor, peripheral nerves during aging.

\section{2 | MATERIALS AND METHODS}

\section{1| Generation and propagation of genetically modified mice}

All animal experiments were carried out in compliance with the protocols approved by the Johns Hopkins University Institutional Animal Care and Use Committee (IACUC). SCspecific MCT1 knockout mice (P0-Cre ${ }^{+/-}, \mathrm{MCT}^{\mathrm{f} / f}$ or noted as $\mathrm{P} 0$-cMCT1 KO mice) and control littermates (P0-Cre ${ }^{-/}, \mathrm{MCT}^{f / f}$ or noted as wild-type [WT]) were generated by crossing MCT1 ${ }^{\mathrm{f} / \mathrm{f}}$ mice to transgenic mice with Cre recombinase driven by melin protein zero (P0) promoter (P0-Cre mice, stock no. 017927, The Jackson Laboratory; Feltri et al., 1999). To generate the MCT1 ${ }^{\mathrm{fff}}$, an original targeting construct for MCT1 exon2, containing ATG translation initiation site, was obtained from the European Conditional Mouse Mutagenesis Program (EUCOMM; Skarnes et al., 2011). 5' arm of the targeting construct was modified for improving the efficiency of homologous recombination. Germline transmission for the chimeric mice, produced by targeting 129 ES cells with the construct, was confirmed by a series of genotyping PCR analyses and several transgenic lines were established. To remove the selection cassette from the targeting construct, the transgenic mice were bred with FLP recombinase deleter mice (Stock 009086; Jackson Laboratory, Bar Harbor ME). The resulting MCT1 ${ }^{f / f}$ mice, harboring conditional/inducible-ready alleles, were back-crossed for two generations with wild-type C57/BL6 mice. For all comparisons, only littermate controls were used. Validation for $\mathrm{MCT} 1{ }^{\mathrm{f} / \mathrm{f}}$ mice was conducted by Southern blot and PCR. Genotypes for MCT1 ${ }^{f / f}$ and P0-cMCT1 KO mice were performed by PCR with the following primer pairs: MCT1 ${ }^{f / f}$ forward, 5'-GCA GCA TGT GGT CCT CTC TTA AG-3', reverse 5'-GTC CTC ACC TCT CTG TGC-3'; MCT1, forward, 5'-GCA GCA TGT GGT CCT CTC TTA AG-3', reverse, 5'-TGG TTC TCT TGT TAT CAG TGT TGG GTG-3'; P0-Cre, forward, 5'-GTG AAA CAG CAT TGC TGT CAC TT-3', reverse, 5'GCG GTC TGG CAG TAA AAA CTA TC-3'. PCR amplification (using Radiant ${ }^{\mathrm{TM}}$ 2x RED Taq Mix; Alkali Scientific) was performed in 39 cycles (for MCT1 ${ }^{\mathrm{f} / f}$ and MCT1) or 35 cycles (for P0-Cre), with each cycle consisting of denaturation at $94^{\circ} \mathrm{C}$ for $30 \mathrm{~s}$, annealing at $52-65^{\circ} \mathrm{C}$ for $30-45 \mathrm{~s}$, depending on the primer pair's annealing temperature, and extension at $72^{\circ} \mathrm{C}$ for $45-60 \mathrm{~s}$.

\subsection{Nerve histology and morphometry}

Deeply anesthetized mice were transcardially perfused with $0.1 \mathrm{M}$ phosphate buffer saline (PBS) followed by $4 \%$ paraformaldehyde fixative and nerves or dorsal/ventral roots of spinal nerves were dissected. Tissue for immunofluorescence staining was post-fixed for $4 \mathrm{hr}$ in $4 \%$ 
paraformaldehyde, cryoprotected in 25\% sucrose and sectioned on a Leica CM1900 cryostat. Sections (20 $\mu \mathrm{m}$ thickness) were immunostained on slides for MCT1 (generated for laboratory [Morrison et al., 2015]; 1:200), S100 (Dako; 1:500), phosphorylated neurofilament (SMI31; Covance; 1:5,000), c-Jun (clone 60A8; Cell Signaling Technology; 1:300) or CD271 (p75 ${ }^{\mathrm{NTR}}$; BioLegend, 1:2,000) either alone or in combination.

Photomicrographs were acquired with ZEN Digital Imaging for LSM 800 (Zeiss). Tissue for electron microscopy or semi-thin nerve histology was post-fixed with $2.5 \%$ glutaraldehyde in $4 \%$ paraformaldehyde for at least 3 days and embedded in Epon 812 resin. Embedded nerves were cut either semi-thin $(1 \mu \mathrm{m})$ and stained with toluidine blue or thin $(70 \mathrm{~nm})$ and stained with citrate/uranyl acetate. Toluidine blue-stained sections were used for quantification of myelinated axon number, myelinated axon diameter, myelin thickness, or $g$ ratio. Our technique is well supported in the literature, as an identical procedure has been followed in a number of publications (Painter et al., 2014; Viader et al., 2013). For each of these histologic features, photomicrographs of nerves or roots were taken on Nikon E800, imported and quantified manually with Zeiss AxioVision 4.8 software. $g$ ratio was calculated as the ratio of the diameter of axons divided by the diameter of myelin sheaths. If more than one fascicle was present in a sample, the largest nerve fascicle was quantified in its entirety. An experimenter, who was blinded to animal genotypes, performed all morphometric analyses.

\subsection{RNA preparation and quantitative real-time reverse transcription-PCR}

Deeply anesthetized mice were transcardially perfused with 0.1 M PBS to remove the blood, and the sciatic nerves or dorsal and ventral roots of spinal nerves were rapidly dissected. RNA was isolated by an RNeasy Mini Kit (Qiagen), reverse transcribed to cDNA with a High Capacity cDNA Reverse Transcription Kit (Applied Biosystems) and quantified by real-time RT PCR using Taqman probes (Applied Biosystems) for MCT1 (Thermo Fisher Scientific; Catalog \# 4351372), MCT2 (Thermo Fisher Scientific; Catalog \# 4331182), MCT4 (Thermo Fisher Scientific; Catalog \# 4331182), GLUT1 (Thermo Fisher Scientific; Catalog \# 4331182), GLUT3 (Thermo Fisher Scientific; Catalog \# 4331182), TRPV1 (Thermo Fisher Scientific; Catalog \# 4448892), or GAPDH (Thermo Fisher Scientific; Catalog \# 4352339E) on a StepOne Plus RT-PCR System (Applied Biosystems).

\section{4| Western blotting}

Peripheral nerves dissected after transcardial perfusion of deeply anesthetized mice with 0.1 M PBS were homogenized in T-PER (Thermo Scientific) and run on Mini-Protean TGX Gels (10\%; Bio-Rad) and transferred to nitrocellulose membranes (Bio-Rad). For all Western blots, 15-30 $\mu \mathrm{g}$ of proteins were separated on the gel. Membranes were incubated overnight with MCT1 (generated for laboratory (Morrison et al., 2015); 1:200), P0 (Aves Labs. Inc.; catalog \# PZO; myelin protein-zero chicken polyclonal anti-peptide antibody; 1:4,000), MBP (Millipore Sigma; catalog \# AB980; anti-myelin basic protein antibody; 1:250), or MAG (Millipore Sigma; catalog \# PA5-30087; polyclonal antibody; 1:500) antibodies and visualized with Amersham ECL Reagant (GE Healthcare) on ImageQuant LAS 4000 (GE Healthcare). After visualizing for above primary antibodies, blots were stripped with Restore Western Blot Stripping Buffer (Thermo Scientific), reprobed overnight 
with $\beta$-actin (Millipore Sigma; catalog \# A5316; monoclonal anti- $\beta$-actin antibody; 1:5,000), and again visualized by ECL reagent, as described above.

\section{5 | Nerve conduction studies}

Electrophysiologic recordings were performed to measure sensory nerve action potentials (SNAPs) from the tail nerve and compound muscle action potentials (CMAPs) by using a Neurosoft-Evidence 3102evo electromyograph system (Schreiber \& Tholen Medizintechnik, Stade, Germany). During all recording sessions, mice were anesthetized with $2 \%$ isoflurane and positioned face down. To accurately localize testing sites for SNAP recordings, a baseline site between the 2nd and 3rd vertebrae (at top/beginning of the tail) was established and electrodes (30G platinum subdermal needle electrodes, Natus Medical, Inc., San Carlos, CA) were placed at $5 \mathrm{~mm}$ and $10 \mathrm{~mm}$ (+/- recording), $20 \mathrm{~mm}$ (ground/reference), $30 \mathrm{~mm}$, $35 \mathrm{~mm}$ (+/- proximal stimulation), $45 \mathrm{~mm}$, and $50 \mathrm{~mm}$ (+/- distal stimulation) from the baseline site. Stimulation of each nerve segment was performed, with increasing voltage, until the maximal response was achieved, as evidenced by no further increase or reduction in SNAP amplitude, despite an increase in stimulation voltage. Response latency for each proximal or distal stimulation was measured from stimulus onset, and peak-to-peak amplitudes were calculated. Sensory NCV was calculated by dividing the distance between the points of proximal and distal stimulations (here, fixed at $15 \mathrm{~mm}$ ) by the difference between their response latencies. The temperature of the tail was measured during each experiment by the Digi-Sense Infrared Thermometer (Model 20250-05). The CMAPs were determined by placing stimulating electrodes $(27 \mathrm{G}$ stainless steel needle electrodes, Natus Medical, Inc., San Carlos, CA) at the sciatic notch and Achilles tendon, and recording electrodes in the lateral plantar muscles of the foot. Similar to the SNAP recordings, stimulation voltage for CMAP recordings was adjusted for maximal response. Nerves were stimulated by very short $(<0.2 \mathrm{~ms})$ electrical impulses. Response latency for each proximal or distal stimulation was measured from stimulus onset, and peak-to-peak amplitudes were calculated. Motor NCV was calculated by dividing the distance between sciatic notch and Achilles tendon by the difference between the response latencies. Unless otherwise indicated, all nerve conduction studies were conducted at room temperature. The investigator performing electrophysiologic recordings was blinded to mice genotypes throughout the study.

\subsection{Measurement of node of Ranvier length}

The sural nerve from aged mice was dissected immediately, desheathed, and dissociated into single fibers by treatment with collagenase $(3.6 \mathrm{mg} / \mathrm{ml}$; Sigma) for $1 \mathrm{hr}$ at room temperature (RT). Fibers were teased apart gently and spread on coverslips that had been coated in a few spots with Cell-Tak (Collaborative Research, Bedford, MA). After teasing, the preparations were fixed in $4 \%$ paraformaldehyde in $0.1 \mathrm{M}$ phosphate buffer, $\mathrm{pH} 7.2$, for $30 \mathrm{~min}$, rinsed in $0.05 \mathrm{M}$ phosphate buffer, $\mathrm{pH}$ 7.4, for $10 \mathrm{~min}$, and air-dried. Teased nerve fibers were immunostained for anti-Caspr antibody (rabbit polyclonal; 1:200; Abcam) and anti-AnkyrinG antibody (mouse monoclonal; 1:200; clone N106/36; NeuroMab). Images were acquired with ZEN Digital Imaging for LSM 800 (Zeiss) and analyzed by using ZEN blue edition software (Zeiss). The node length was measured without correcting for fiber shrinkage during fixation. 


\section{7 | Intra-epidermal nerve fiber analysis}

Analysis of footpads for intra-epidermal nerve fiber density (IENFD) was performed as previously described (Chiorazzi et al., 2018). Briefly, footpads were dissected out and were washed in $0.1 \mathrm{M}$ phosphate buffer and placed in cryoprotectant solution (30\% glycerol). Tissue blocks were sectioned by freezing microtome at $50 \mu \mathrm{m}$ intervals and immunohistochemical staining was performed for protein gene product 9.5 (PGP 9.5; rabbit polyclonal; AbD Serotec, a Bio-Rad Company, Kidlington, UK) using a free-floating protocol. The total number of PGP 9.5-positive IENF in each section was counted, the length of the epidermis was measured and the linear density of IENF/mm was obtained. An experimenter, who was blinded to animal genotype, performed the tissue processing, immunostaining, and analysis.

\section{8 | Behavioral studies}

An experimenter blinded to animal genotype carried out all behavioral tests. One day before behavioral testing, mice were acclimated to the environment for $1 \mathrm{hr}$. Mechanical sensitivity was assessed with the von Frey test by the frequency method. Two calibrated von Frey monofilaments (low force, $0.07 \mathrm{~g}$; high force, $0.45 \mathrm{~g}$ ) were used. Each von Frey filament was applied perpendicularly to the plantar side of hind paw for $\sim 1 \mathrm{~s}$; the stimulation was repeated 10 times to the paw. The occurrence of paw withdrawal in each of these 10 trials was expressed as a percent response frequency: paw withdrawal frequency $=$ (number of paw withdrawals $/ 10$ trials) $\times 100 \%$. Mean of the left and the right hindpaw measurements from each mouse were calculated. For the Hargreaves test, mice were placed under a transparent plastic box $(4.5 \mathrm{~cm} \times 5 \mathrm{~cm} \times 10 \mathrm{~cm})$ on a glass floor. Infrared light was delivered through the glass floor to the hind paw. After acclimatization sessions, the latency for the animal to withdraw its hind paw was measured.

\subsection{SC culture and Seahorse bioenergetic analysis}

Cultures of SCs were prepared from the sciatic nerves of 4-week-old mice as described previously (Scheib \& Hoke, 2016) with slight modifications. Briefly, dissected sciatic nerves were cut into small pieces and dissociated at $37^{\circ} \mathrm{C}$ in $0.3 \%$ collagenase (Gibco) for $45 \mathrm{~min}$ and $0.25 \%$ trypsin for $15 \mathrm{~min}$. Dissociated cells were resuspended in SC media (ScienceCell), which consists of basal media supplemented with 5\% FBS, 1\% SC growth supplement and $1 \%$ penicillin/streptomycin solution, and plated. On Day 1 after plating, the media was switched to SC purification media, which consists of SC media supplemented with $10 \mu \mathrm{M}$ arabinosylcytosine (AraC, Millipore Sigma), for 2 days. The media was then switched to SC media for 2 days, which was followed by switching to SC purification media for the next 2 days. SCs purity (greater than 95\%) was confirmed by immunostaining with antibody against S100 and was used for measurements of oxygen consumption and extracellular acidification using XF96 extracellular flux analyzer (Seahorse Bioscience, North Billerica, MA; Yang et al., 2013) following the manufacturer's instructions. SCs were grown in the Seahorse XF96 cell culture microplate at 10,000 cells/well. Bioenergetic analysis was performed by sequentially injecting $2 \mu \mathrm{M}$ oligomycin, $4 \mu \mathrm{M}$ carbonyl cyanide $p$-(trifluoromethoxy) phenylhydrazone (FCCP), $0.5 \mu \mathrm{M}$ rotenone and $4 \mu \mathrm{M}$ antimycin. Data 
are expressed as oxygen consumption rate (OCR) in picomole per minute and extracellular acidification rate (ECAR) in milli pH per minute for 10,000 cells.

\subsection{Nerve lipidomics}

Sural nerves from aged ( 8 months old) mice were dissected, cleaned from surrounding fat and connective tissue, and snap frozen using dry ice. Nerve tissues were extracted using a modified Bligh and Dyer procedure to obtain a crude lipid fraction (Bligh \& Dyer, 1959). In brief, tissues were homogenized in $\mathrm{ddH}_{2} 0$ and protein normalized volumes of tissue

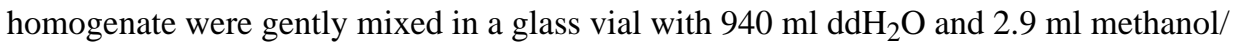
dichloromethane $(2: 0.9, \mathrm{v} / \mathrm{v})$ containing the following internal standards: N-lauroyl-Derythro-sphingosine (Cer d18:1/12:0, $6 \mathrm{ng} / \mathrm{ml}$ ), 1,3(d5)-dihexadecanoyl-glycerol (d5-DAG d16:0/16:0, $12.5 \mathrm{ng} / \mathrm{ml}$ ), D-galactosyl- $\beta-1,1$ ' N-lauroyl-D-erythro-sphingosine (GlcCer d18:1/12:0, $3.3 \mathrm{ng} / \mathrm{ml}$ ), D-lactosyl- $\beta$-1,1' N-lauroyl-D-erythro-sphingosine (LacCer 18:1/12:0, $10.6 \mathrm{ng} / \mathrm{ml}), 1,3(\mathrm{~d} 5)$-dihexadecanoyl-2-octadecanoyl-glycerol (D-5 TAG 16:0/18:0/16:0, $0.5 \mathrm{ng} / \mathrm{ml}$ ), cholesteryl-d7 palmitate (cholesteryl-d7 ester 16:0, $30 \mathrm{ng} / \mathrm{ml}$ ), 1,2-dilauroyl-sn-glycero-3-phosphate (sodium salt; PA d12:0/12:0, 1,025 ng/ml), 1,2dilauroyl-sn-glycero-3-phosphocholine (PC 12:0/12:0, $0.2 \mathrm{ng} / \mathrm{ml}$ ), 1,2-dilauroyl-snglycero-3-phosphoethanolamine (PE d12:0/12:0, $1.6 \mathrm{ng} / \mathrm{ml}$ ), 1,2-dilauroyl-sn-glycero-3phospho-[1'-rac-glycerol] (PG d12:0/12:0, $200 \mathrm{ng} / \mathrm{ml}$ ), 1,2-dilauroyl-sn-glycero-3-phosphoL-serine (PS d12:0/12:0), and N-lauroyl-D-erythro-sphingosylphosphorylcholine (SM d18:1/12:0, $0.3 \mathrm{ng} / \mathrm{ml})$. All internal standards were purchased from Avanti Polar Lipids, Inc. (Alabaster, AL). To obtain a biphasic mixture, an additional $1 \mathrm{ml}$ of $\mathrm{ddH}_{2} \mathrm{O}$ and $0.9 \mathrm{ml}$ dichloromethane was added and vortexed. The resultant mixture was incubated on ice for 30 min and centrifuged $\left(10 \mathrm{~min}, 3,000 \mathrm{~g}, 4^{\circ} \mathrm{C}\right)$ to separate the organic and aqueous phases. The organic phase was removed and stored at $-20^{\circ} \mathrm{C}$. Just prior to analysis, $1 \mathrm{ml}$ of the organic layer was dried using a nitrogen evaporator (Organomation Associates, Inc., Berlin, MA) and re-suspended in $250 \mu \mathrm{l}$ of running solvent (dichloromethane: methanol [1:1] containing $5 \mathrm{mM}$ ammonium acetate), and $5 \mathrm{mg} / \mathrm{ml}$ of ceramide C17:0 used to track instrument performance. All solvents used were HPLC grade. Samples were analyzed on a 5600 Triple TOFTM mass spectrometer (AB Sciex, Concord, Canada). For data analysis, lipid peak intensities were first log transformed to achieve a normal distribution. They were then filtered based on the following criteria: skewness $<10$, kurtosis $<10$, and IQR:SD ratio differing less than $50 \%$ from 1.35 (Rice, 2007). Using these criteria 38 of the 755 lipid analytes were excluded from further analyses. Log-transformed lipid intensities were investigated in a univariate analysis, and the means between the two groups were compared using $t$ tests with a Benjamini-Hochberg correction (Benjamini \& Hochberg, 1995) for multiple comparisons (MathWorks, 2018). For visualization, lipid species were Z-scored to have a mean of 0 and a SD of 1 . Principal component analysis (PCA) was used to interrogate variations in the lipidomic data. This procedure creates linear combinations of the original variables to create principal components (Jolliffe, 2011) in an unsupervised manner. An overall PCA, which was limited to three components, was conducted on the entire data set. Analysis was conducted using R version 3.5.1 with mixOmics version 3.8 (Rohart, Gautier, Singh, \& Le Cao, 2017). 


\subsection{Quantification and statistical analysis}

Analyses were performed blinded to animal genotype and treatment. Although we did not perform statistical tests to predetermine sample size, our samples sizes are similar to previously published studies in the field. Statistical analyses were performed with GraphPad Prism 8 (GraphPad Software) by using unpaired $t$ test with two tails with unequal variance, one-way ANOVA, or two-way ANOVA with post hoc test when required conditions were met. The number of animals per group or independent repeats $(n)$, the statistical test used for comparison, and the statistical significance ( $p$ value) was stated for each figure panel in the respective legend. All data were presented as the mean $\pm S E M$ unless otherwise noted. Differences in the $p$ values of $<0.05$ were considered statistically significant.

\subsection{Data sharing}

The data that support the findings of this study are available from the corresponding author upon reasonable request.

\section{3| RESULTS}

\subsection{Deletion of MCT1 from SCs reduces its expression in sciatic nerves without any compensatory alterations in expression of other metabolic mediators}

With the goal of exploring the role of SC-specific MCT1 in peripheral nerve biology during development and aging, we produced mice with SC-selective ablation of MCT1 (P0-cMCT1 $\mathrm{KO}$ ) by mating MCT1 $1^{f / f}$ mice with P0-Cre mice (Figure 1a). P0-Cre mice express Crerecombinase in both myelinating and non-myelinating SCs without expression in other cells of the peripheral nerve or in oligodendroglia (Feltri et al., 1999). Since MCT1 is expressed in other cells of the peripheral nerve and oligodendroglia (Lee et al., 2012), cell-selective knockout is necessary for defining the function of SC MCT1 on peripheral nerve biology. Photomicrographs of this $\mathrm{P} 0$-Cre line mated to YFP reporter mice demonstrate selective expression in SCs (Figure 1b), as published previously (Feltri et al., 1999). We also confirmed that the $\mathrm{P} 0$-Cre is not itself detrimental for nerve function by measuring sensory and motor nerve conduction velocities (NCV) and tail sensory (sensory nerve action potential [SNAP]), and sciatic nerve motor (compound muscle action potential [CMAP]) amplitudes from $\mathrm{P} 0-\mathrm{Cre}^{+/}$, MCT1 ${ }^{w t / W t}$ mice (Supporting Information Figure S1a-d). We evaluated mice of each genotype for knockout of MCT1 at the levels of protein, by immunofluorescence (Figure 1c) and Western blot (Figure 1d and Supporting Information Figure S8), and mRNA by real-time RT PCR (Figure 1e). Immunofluorescence clearly shows loss of MCT1 from SCs in the endoneurium in P0-cMCT1 KO mice (Figure 1c; arrows) without loss of MCT1 from perineurial cells (Figure 1c; arrowheads). Ablation of MCT1 in SCs was enough to reduce its expression significantly in whole sciatic nerve (Figure 1d-e), suggesting that SCs express relatively high amounts of MCT1 compared to other cells in the peripheral nerve. Interestingly, SC-specific MCT1 deletion did not cause any compensatory alterations in the expression of other metabolic transporters, MCT2, MCT4, GLUT1, and GLUT3, in sciatic nerves (Figure 1f-i). 


\subsection{Sensory, but not motor, nerve conduction velocity decreases in maturing P0-cMCT1 KO mice}

We measured sensory and motor NCVs and SNAP and CMAP amplitudes to assess peripheral nerve health and axonal integrity during aging following SC-specific MCT1 deletion. Assessment of NCV is an excellent measure of the myelination state of nerves, and SNAP and CMAP amplitudes are reliable indicators of axonal integrity. Although NCV declines with age in humans (Di Iorio et al., 2006), NCV does not decline in male and female mice prior to 20 months of age (Walsh et al., 2015). Moreover, tail SNAP amplitude remains constant over the life span of female mice (Walsh et al., 2015). We evaluated nerve function, by repeated electrophysiologic testing, in P0-cMCT1 KO and littermate control female mice at room temperature, where the tail temperature was usually recorded to be between 22 and $24^{\circ} \mathrm{C}$ (Figure 2). Tail temperature of 4-month-old mice measured at the beginning of electrophysiologic recording was $23.20 \pm 0.12^{\circ} \mathrm{C}$ for wild-type and $23.59 \pm$ $0.58^{\circ} \mathrm{C}$ for $\mathrm{P} 0$-cMCT1 KO (Mean $\pm S E M, n=5$ for wild-type and 8 for P0-cMCT1 KO; $p$ $=.533$, unpaired $t$ test). At 2 months of age, there were no alterations in sensory and motor NCVs (Figure 2a,c) or SNAP and CMAP amplitudes (Figure 2b,d) following SC-specific MCT1 deletion, suggesting that SC-specific MCT1 has no critical role during this stage of peripheral nerve development. At 4 months of age, however, sensory (Figure 2a), but surprisingly not motor (Figure 2c), NCV was significantly decreased in mice lacking MCT1 in SCs. The discrepancy in sensory NCV due to MCT1 ablation in SCs was persistent during maturation (3-6 months) and aging (10-12 months) of mice. The decreased sensory NCV and unchanged SNAP amplitude due to MCT1 deletion in SCs at 4 months of age was also confirmed by electrophysiologic recordings performed while maintaining tail temperature at $32-34^{\circ} \mathrm{C}$ (Supporting Information - Figure S2a,b). We have also confirmed that the decrease in sensory NCV during aging in female mice is evident in littermate male mice having SCspecific MCT1 deficiency, suggesting that the electrophysiologic deficit observed due to MCT1 deficiency in SCs is independent of gender (Supporting Information Figure S3a,b). These findings suggest that MCT1 in SCs is crucial for maintenance of sensory nerve function during maturation and aging.

\subsection{SC-specific MCT1 deletion results in mild sensory nerve hypomyelination during aging}

The slower sensory NCV in mice with SC ablation of MCT1 compared with littermate control mice led us to investigate sural nerve (purely sensory) morphology in early postnatal (14-day-old; Figure 3a-d and Supporting Information Figure S4a-c), developing (2-monthold; Figure 3e-h and Supporting Information Figure S4d-f), maturing (4-month-old; Figure $3 \mathrm{i}-\mathrm{l}$ and Supporting Information Figure S4g-i), and aging (12-month-old; Figure 3m-p and Supporting Information - Figure S4j-1) mice. Consistent with the electrophysiologic recordings of sensory NCV and SNAP amplitudes, we observed a remarkable correlation between genotype and age with respect to myelination and myelinated axon counts. Mice with no MCT1 in SCs developed normally, showed no anatomical difference in term of myelinated axon number, myelination, and myelinated axon caliber at the ages of 14 days and 2 months compared with their littermate control mice (Figure 3a-h and Supporting Information Figure $\mathrm{S} 4 \mathrm{a}-\mathrm{f}$ ). By 4 months of age, however, the $g$ ratio, which reflects the degree of axonal myelination (axon diameter divided by axon plus myelin diameter), for 
sural nerve axons was significantly increased during maturation, indicating myelin thinning, without any alteration in the number of myelinated axons (Figure 3k). The sensory axon hypomyelination in mice having SC-specific MCT1 deficiency was further confirmed by decreased myelin thickness without alterations in axon diameter (Supporting Information Figure S4g-i). Identical to electrophysiology results (Figure 2), the hypomyelination by $g$ ratio and myelin thickness was present at 12 months, as well (Figure 3o,p and Supporting Information Figure S4j-1), indicating the crucial role of SC-specific MCT1 in sensory axon myelin maintenance during maturation and aging. For the 4 and 12-month-old sural nerves, the scatter plot of the $g$ ratio against the axon diameter revealed that the increased $g$ ratio for sensory axons was most prominent for axons with smaller diameters (Figure 31,p).

Furthermore, mice with no MCT1 in SCs showed decreased myelinated axon counts during aging that was present by 12 months of age (Figure $3 \mathrm{n}$ ). This was likely due to reduced myelination rather than loss of axons since there was no loss of axons when quantifying all axons greater than $1 \mu \mathrm{m}$ by electron microscopy. The density of axons per $1,000 \mu \mathrm{m}^{2}$ were $21.74 \pm 1.73$ and $21.98 \pm 2.11$ for wild-type and P0-cMCT1 KO mice, respectively (11-14 random electron micrographs showing a total of 88-126 axons per mouse sural nerve captured at $\times 5,000$ magnification were analyzed for each mouse, Mean $\pm S E M, n=3$ per group; $p=.935$, unpaired $t$ test). Though both myelinating and non-myelinating SCs express the P0 promoter (Cheng \& Mudge, 1996; Georgiou \& Charlton, 1999), we did not see any morphologic abnormalities in Remak bundle organization at any age due to non-myelinating SC MCT1 ablation (Figure 3, left panel's electron micrographs). Besides the overall hypomyelination, alterations in the length of nodes of Ranvier along axons can also contribute to overall NCV. In aged mice, the node length, as measured by distance between paranodes (distance between two Caspr clusters), was moderately, but significantly, increased in sural nerves from P0-cMCT1 KO mice compared to wild-type mice (Supporting Information Figure S5a,b). The expansion in nodal length increases nodal capacitance and slows intra-axial current flow from the node into the internodes. Given the otherwise normal appearance of nodal and paranodal proteins, we suspect that the nodal length increase is likely another reflection of hypomyelination. These findings demonstrate that MCT1 in SCs is important for preservation of sensory axon myelin during aging.

The differential sensory and motor electrophysiologic features and histologic alterations in sural nerve due to SC-specific MCT1 deletion was further confirmed by quantitative assessment of dorsal and ventral roots of the lumbar region of the spinal cord (Figure 4 and Supporting Information Figure S6). Dorsal roots are composed of purely sensory axons, while ventral roots are composed of purely motor axons. Quantitative morphometry of toluidine blue-stained micrographs revealed no alteration in myelinated axon counts and caliber at 12 months of age of both the dorsal and ventral roots due to MCT1 deficiency in SCs (Figure 4a,b,e,f and Supporting Information Figure S6a-f). However, SC-specific MCT1 deficiency increased the $g$ ratio for the dorsal (Figure 4c,d), but not ventral (Figure $4 \mathrm{~g}, \mathrm{~h}$ ), roots of spinal nerve at 12 months of age, which again appears to primarily affect the smallest myelinated fibers. The reduced myelin thickness of dorsal roots thus confirms the hypomyelination observed more distally in the purely sensory sural nerve. 


\subsection{Vulnerability of sensory axons is not due to differential expression of MCT1 in sensory nerves compared to motor nerves}

The selective impact of reducing SC MCT1 on sensory nerves, with sparing of motor nerves, led us to speculate that there may be differential expression of MCT1 in SCs associated with sensory and motor nerves. To address this issue, we measured the expression of MCT1 mRNA in purely sensory dorsal roots and purely motor ventral roots of spinal nerves. Interestingly, no difference in MCT1 mRNA expression was detected between dorsal and ventral roots (Supporting Information Figure S6g), suggesting that the vulnerability of sensory, but not motor, nerves is not due to differential expression of MCT1.

\subsection{Mice with SC MCT1 deletion develop decreased mechanical sensitivity during aging}

Impaired sensory nerve electrophysiology and histology in mice with SC-specific MCT1 deletion led us to investigate if there were sensory deficits in these mice. The behavioral responses to repetitive punctate mechanical stimulation and noxious thermal stimulation were assessed with the von Frey test by the frequency method and the Hargreaves test, respectively (Figure 5). Mice with or without SC MCT1 deletion revealed no difference in mechanical and thermal sensitives during development and maturation (2 and 4 months of age, respectively; Figure 5a-c). However, mice with SC MCT1 ablation had decreased mechanical sensitivity during aging compared with littermate control mice, as indicated by decreased paw withdrawal frequency to repetitive high force $(0.45 \mathrm{~g})$ von Frey filament stimulation (Figure 5b), at 12 months of age. The increased paw withdrawal frequency to repetitive high force mechanical stimulation in 12 months wild-type mice is consistent with a recent report suggesting an elevated sensitivity to mechanical stimuli with aging (Weyer et al., 2016). In contrast, no alteration was observed in paw withdrawal latency to noxious thermal stimulation at any age (Figure 5c). The decrease in mechanical, and not thermal, sensitivity is interesting and expected as axons conveying thermal signals are unmyelinated. Furthermore, the expression of transient receptor potential vanilloid type 1 (TRPV1), which behaves as a molecular integrator of chemical and thermal noxious stimuli in the PNS determining nociceptive responses, was unchanged in the sciatic nerve following SC-specific deletion of MCT1 (Figure 5d). The unaltered thermal sensitivity in aging mice was further supported by unchanged intra-epidermal nerve fiber density (IENFD), as measured by PGP9.5-immunoreactive nerve counts normalized to epidermal area, which measures unmyelinated nociceptive nerve fibers in the skin (Figure 5e). These findings suggest that SC-specific MCT1 is critical for maintenance of mechanical, but not heat, sensitivity during aging.

\subsection{Selective ablation of MCT1 impairs glycolytic and mitochondrial functions in SCs}

In order to begin to understand how MCT1 deficient SCs have impaired capacity for myelination, their capacity for oxidative metabolism and glycolysis was measured by quantifying the rate of real-time oxygen consumption (OCR; Figure 6a) and extracellular acidification (ECAR; Figure 6b), respectively, in a live cell assay with the Seahorse extracellular flux analyzer. Given the reduction of the lactate transporter MCT1, which is necessary for transporting lactate produced from glycolysis, it was not surprising that ECAR was reduced. Oligomycin-induced ECAR, an indicator of glycolytic activity, was 
significantly decreased by MCT1 deficiency in SCs, while ECAR during basal respiration was not changed (Figure 6c). Remarkably, both basal oxygen consumption and uncoupled respiration (maximal mitochondrial oxygen consumption capacity following the addition of FCCP, which mimics a physiologic "energy demand") were significantly reduced in cultured SCs with MCT1 deficiency (Figure 6d). Spare respiratory capacity (SRC), which is defined as the difference between maximal respiration and basal respiration, is a measure of the ability of the cell to respond to increased energy demand as well as how closely the cell is to respiring at its theoretical maximum. SRC was significantly decreased due to MCT1 deficiency in cultured SCs (Figure 6e). These findings demonstrate that MCT1 deficiency impairs glycolytic and mitochondrial functions of SCs and worsens their fitness or flexibility to respond to stress stimuli and/or metabolic demands.

\subsection{Hypomyelination due to MCT1 deficiency is associated with deficits in synthesis of MAG, but not other myelin proteins, reversion of SC to less mature state, and impaired lipogenesis in peripheral nerves}

Several structural proteins play a role in the maintenance of myelin in peripheral nerves. To begin to investigate potential mechanisms for nerve hypomyelination with aging, expression of myelin proteins was investigated in peripheral nerves from aged wild-type and P0-cMCT1 $\mathrm{KO}$ mice. Interestingly, expression of major peripheral myelin proteins, myelin protein zero (P0) and myelin basic protein (MBP), were unchanged in sciatic nerves with no MCT1 in SCs (Figure 7a,b and Supporting Information Figure S8). In contrast, there was significantly decreased expression of myelin-associated glycoprotein (MAG) from peripheral nerves lacking SC MCT1 (Figure 7c and Supporting Information Figure S8). MAG in peripheral nerve is important for long-term stabilization of myelinated axons and prevents axonal degeneration both in vitro and in vivo (Nguyen et al., 2009). These findings suggest that MCT1 in SCs contributes to the synthesis of MAG, but not MBP or P0, during aging.

The described glycolytic and mitochondrial dysfunction in MCT1-deficient cultured SCs, led us to investigate potential developmental errors in SCs without MCT1. Interestingly, cJun expression was dramatically upregulated in sciatic nerves from maturing mutant mice (Figure 7d,e). The upregulation of c-Jun, which is a master regulator of SC differentiation (Sasaki, Hackett, Kim, Strickland, \& Milbrandt, 2018), in sciatic nerves indicates that MCT1 deficiency causes SCs to arrest at a less mature developmental state. Since the upregulation of c-Jun is also an indicator of mitochondrial stress response (Qureshi, Haynes, \& Pellegrino, 2017), the immaturity of SCs due to MCT1 deficiency was further confirmed by upregulated expression of p75-neurotrophin receptor (p75 $\left.{ }^{\mathrm{NTR}}\right)$, a prototype marker for immature SCs (Chen, Yu, \& Strickland, 2007; Jessen \& Mirsky, 2008), in sciatic nerves (Supporting Information Figure S7).

Myelin is composed of myelin proteins and various lipid species. Given that the two major structural myelin proteins in peripheral nerves, $\mathrm{MBP}$ and $\mathrm{P} 0$, were unaltered in hypomyelinated nerves from P0-cMCT1 KO mice, the most likely explanation for hypomyelination was reduced synthesis or increased metabolism of SC lipids that are critical components of myelin sheaths. We, therefore, quantified, in an unbiased manner, the abundances of individual lipids from multiple classes in sural nerves from aged mice to 
explore the influence of SC-specific MCT1 deficiency in lipid metabolism. We detected a total of 717 lipid species by mass spectrometry consisting of 2 cholesterol esters (CE), 8 ceramides (CER), 66 diacylglycerides (DAG), 3 dihexosylceramides (Hex3Cer), 15 hexosylceramides (HexCer), 5 lysophosphatidylcholines (LPC), 2 lysophosphotidylserines (LPS), 1 phosphatidic acid (PA), 50 phosphatidylcholines (PC), 56 phosphatidylethanolamines (PE), 6 phosphatidylglycerols (PG), 51 phosphatidylserines (PS), 18 sphingomyelins (SM), and 433 triacylglycerides (TAG). In univariate analyses, we found that the peak intensities for 382 lipids were significantly different in sural nerves obtained from SC-specific MCT1 deficient mice compared to littermate wild-type mice, and 322 of these lipids remained significant after correction for multiple comparisons (Figure $7 \mathrm{f}$ and Supporting Information Table S1). These lipids consisted of 4 DAGs, 5 SMs, and 313 TAGs. By principal component analysis, the overall PCA explained a cumulative $82.5 \%$ of the total variance (with three components), and $58.2 \%$ of this variance was explained by the first component. There was a visual separation between the lipidomic profiles of sural nerves from wild-type and P0-cMCT1 KO mice in the first three components of the overall PCA (Figure $7 \mathrm{~g}$ ), indicating that loss of myelin from sensory nerves of mutant mice with SCspecific MCT1 deletion is associated with impaired production or increased metabolism of peripheral nerve lipids, especially triacylglycerides, diacylglycerides, and sphingomyelins.

\section{4 | DISCUSSION}

Our laboratory used a newly generated conditional MCT1 knockout mouse to produce mice lacking MCT1 in their SCs (i.e., P0-cMCT1 KO mice). To our surprise, MCT1 deletion in SCs led to no compensatory alterations in the expression of other MCTs and GLUTs, and the mice develop normally with no abnormalities in peripheral nerves through 2 months of age. By 4 months of age; however, P0-cMCT1 KO mice develop reduced sensory, but not motor, NCV that persists through at least 12 months of age and is associated with a corresponding reduction in myelin thickness and increase in the length of nodes of Ranvier. Myelin thickness and nodes of Ranvier length are the primary structural determinants of the conduction velocity in myelinated axons (Arancibia-Carcamo et al., 2017). This persistent demyelination is associated with impaired sensory function, as measured by mechanical sensitivity, by 12 months of age. These results suggest a functional role of SC MCT1 in sensory nerve myelination and axon biology during aging. Though demonstrated by both electrophysiology and histology, the alterations in myelin are rather small, suggesting that there are redundant energy pathways in SCs that are able to compensate for loss of MCT1 to produce myelin.

SC metabolism is critical for the regulation and maintenance of peripheral nerve development and function, and SCs are the primary cell type that metabolically support PNS axons (Beirowski et al., 2014; Jha \& Morrison, 2018). Emerging evidence documents a close and regulated metabolic interaction between SCs and neurons in the PNS (Fields, 2015; Jha \& Morrison, 2018). GLUTs in endothelial cells (Froehner, Davies, Baldwin, \& Lienhard, 1988), perineurial cells (Gerhart \& Drewes, 1990; Takebe et al., 2008), and SCs (Magnani et al., 1996) facilitate the uptake of circulating glucose into SCs that can be metabolized to pyruvate, lactate, and other energy intermediates. Our findings show that MCT1 deficiency dramatically alters the bioenergetics of SCs. Given the absence of their primary lactate 
transporter, it is not surprising that there was a significant decrease in oligomycin-induced ECAR in cultured mutant SCs, indicating impaired glycolytic activity. Interestingly, absence of SC MCT1 limited not only glycolysis, but mitochondrial function, as well. The reason for mitochondrial dysfunction in SCs lacking MCT1 is not clear, but may be due to lactate accumulation and reduced intracellular $\mathrm{pH}$ in MCT1-deficient SCs. It should be noted, however, that these metabolic studies were conducted in SC cultures and the toxicity of MCT1 ablation may be accentuated in vitro due to specific culture conditions. Perhaps surprisingly, we did not observe any deficits in axonal function or survival, suggesting that ablating MCT1 from SCs does not impair metabolic support for axons. SCs express other lactate transporters, and perhaps these other transporters are capable of maintaining support to axons. Future studies could address this possibility by ablating multiple lactate transporters from SCs.

Sciatic nerves from maturing P0-MCT1 KO mice have increased expression of both c-Jun and $\mathrm{p} 75^{\mathrm{NTR}}$. c-Jun is a negative regulator of SC differentiation, myelination, and has been implicated in demyelinating neuropathies (Jessen \& Mirsky, 2008). p75 ${ }^{\mathrm{NTR}}$, which has been implicated in the regulation of gene expression, cell cycle transitions, and myelination by recruiting specific binding proteins, is a marker for immature SCs that is highly expressed primarily during development and after nerve injury (Cragnolini \& Friedman, 2008; Wang et al., 2017). Though c-Jun is also upregulated by mitochondrial injury, the upregulation of p $75^{\mathrm{NTR}}$ confirms the presence of increased immature SCs in P0-MCT1 KO mice. Interestingly, there is a greater increase in c-Jun-positive SCs, as compared to $\mathrm{p} 75^{\mathrm{NTR}}$ positive SCs, suggesting the presence of mitochondrial injury in SCs in vivo. The importance of upregulation of c-Jun and p $75^{\mathrm{NTR}}$ is twofold. First, the upregulation of these proteins suggest that the deficits in myelination are not merely due to "sick" cells with diminished overall metabolism, but rather a shift in the phenotype of these cells to a more premature state. Second, upregulation of c-Jun and demyelinating neuropathy was also recently reported following dysregulation of $\mathrm{NAD}^{+}$biosynthesis, a key cellular factor in glycolytic metabolism (Verdin, 2015). SC-specific loss of MCT1 may similarly mediate cJun expression through impairment of glycolysis. Though c-Jun and p75 ${ }^{\mathrm{NTR}}$ can also be upregulated by nerve injury, this is unlikely the cause in our mice since c-Jun upregulation occurs prior to any evidence of axon injury.

Myelin membranes have a very high lipid-to-protein ratio, in which lipids constitute 70-80\% of myelin (O'Brien, Sampson, \& Stern, 1967). Lipid biosynthesis is thus critical for SCs during both developmental myelination and maintenance of myelin sheaths during maturation and aging; and abnormalities of lipid metabolism can directly cause hypomyelination (Montani et al., 2018; Schmitt, Castelvetri, \& Simons, 2015). Though the two most abundant myelin proteins, $\mathrm{P} 0$ and MBP, were present in normal amounts, several lipids, including sphingomyelin, triacylyglycerides, and diacylglycerides were reduced in sensory peripheral nerves lacking SC MCT1. This finding illustrates that SC-specific MCT1 deletion does not significantly affect transcription, translation, or stability of at least the most prominent myelin proteins in peripheral nerves, rather hypomyelination appears to be due to reduced production or increased scavenging of myelin lipids. Sphingomyelin is a major component of peripheral nerve myelin, where it represents $10-35 \%$ of the total lipids in mice (Garbay, Heape, Sargueil, \& Cassagne, 2000). Degradation of sphingomyelin is 
reported following peripheral nerve injury (Patti et al., 2012) and chemotherapy-induced peripheral neuropathy (Stockstill et al., 2018); and sphingomyelin content of myelinating DRG cultures correlates with state of demyelination following forskolin treatment (Capodivento et al., 2017). Triacylglycerides are lipids with very short half-lives used primarily for energy production or storage. Though not a component of myelin membranes, themselves, their turnover is quite rapid and they serve as intermediates in the synthesis of longer-chain fatty acids and sphingosine, which are major components of myelin (O'Brien et al., 1967). Interestingly, vitamin B12 deficiency, which causes axonopathy in both spinal cord descending tracts and peripheral nerves (Stabler, 2013), appears to be at least partly dependent on triacylglycerides. Rats on a vitamin B12 deficient diet developed a marked decrease in sciatic nerve triacylglycerides without alterations in other lipids (Turner \& Vevallos, 1968). Diacylglyceride is a precursor for triacylglycerides and plays an important role in lipid biosynthesis. In addition, diacylglyceride is itself an important membrane component and key second messenger in multiple cellular signaling cascades (Carrasco \& Merida, 2007). Thus, the significant reductions in sphingomyelin, triacylglyceride, and diacylglyceride in P0-cMCT1 KO mice may contribute to the age-dependent demyelination and axon loss in sensory nerves of P0-cMCT1 KO mice. Our hypothesis is that impaired glycolytic and oxidative metabolism in SCs leads to increased utilization and impaired synthesis of SC lipids, thus removing a critical substrate for the maintenance of myelin lipids. Though not previously investigated following abnormalities in MCT1, mitochondrial defects caused reduced pyruvate oxidation, aberrant SC lipid metabolism, and peripheral nerve injury (Riviere et al., 2009); and fatty acid synthesis in SCs is necessary for myelination (Montani et al., 2018). Thus, the observed reduction in mitochondrial oxidative phosphorylation in MCT1-deficient SCs is likely one mechanism for aberrant lipid metabolism. Additionally, reduced glycolysis in MCT1-deficient SCs, by lactate retention reducing glucose uptake and glycolysis and impeding intracellular energy production (Leite et al., 2011), will also reduce the availability of pyruvate for oxidation and fatty acid synthesis, potentially contributing to demyelination.

SC-specific MCT1 ablation causes deficits in sensory, but not in motor, myelination of peripheral nerves by 4 months of age, as quantified by electrophysiology and histology; and sensory behavioral deficits by 12 months of age. The selective impact on sensory nerves was not due to differential expression of MCT1, evaluated at the level of mRNA, in sensory versus motor SCs. Though the reason for sensory SCs vulnerability remains unknown, emerging evidence shows that SCs associated with sensory or motor nerves have differential functions and distinct molecular phenotypes (Brushart et al., 2013; Hoke et al., 2006; Wright et al., 2014). Our findings add to these phenotypic differences, suggesting that metabolic signatures or susceptibility to metabolic alterations can also differentiate sensory and motor SCs. Interestingly, peripheral neuropathies, particularly those caused by metabolic dysfunction (i.e., diabetes) or toxins, are most commonly sensory predominant (Katona \& Weis, 2017). Whether alterations in MCT1 or other metabolic transporters are responsible for this vulnerability has not yet been determined.

Our results indicate that SC MCT1 contributes to maintenance of myelin of sensory, but not motor, nerves during aging. Our study details several specific cellular pathways disrupted by deletion of SC MCT1, including MAG expression, SC maturation as reflected by c-Jun and 
p75 ${ }^{\mathrm{NTR}}$ expression, and lipid metabolism, which may contribute to hypomyelination and dysfunction of sensory peripheral nerves (Figure 8). Further studies manipulating this energetic pathway may reveal potential novel treatments for peripheral neuropathies, many of which are extremely common and currently untreatable.

\section{Supplementary Material}

Refer to Web version on PubMed Central for supplementary material.

\section{ACKNOWLEDGMENTS}

The authors would like to thank Dr. Mohamed Farah, Ms. Carol Cooke, Ms. Kimberly Brown, and the Johns Hopkins Neurology Electron Microscopy Core, for their assistance in processing and photographing electron microscopic pictures and processing embedded nerve tissue for toluidine blue staining. We also thank Dr. Ru-ching Hsia and the University of Maryland Electron Microscopy Core Imaging Facility for assistance with photographing electron microscopic pictures. We would also like to thank the Pain Research Core funded by the Blaustein Fund and the Neurosurgery Pain Research Institute at the Johns Hopkins University for providing facilities for behavioral studies. Financial support was provided by NIH-NS086818-01 (B.M.M.).

\section{REFERENCES}

Arancibia-Carcamo IL, Ford MC, Cossell L, Ishida K, Tohyama K, \& Attwell D (2017). Node of Ranvier length as a potential regulator of myelinated axon conduction speed. Elife, 6, 1-15. 10.7554/eLife. 23329

Beirowski B, Babetto E, Golden JP, Chen YJ, Yang K, Gross RW,... Milbrandt J (2014). Metabolic regulator LKB1 is crucial for Schwann cell-mediated axon maintenance. Nature Neuroscience, 17 (10), 1351-1361. 10.1038/nn.3809 [PubMed: 25195104]

Benjamini Y, \& Hochberg Y (1995). Controlling the false discovery rate-A practical and powerful approach to multiple testing. Journal of the Royal Statistical Society Series B-Methodological, 57(1), 289-300.

Bligh EG, \& Dyer WJ (1959). A rapid method of total lipid extraction and purification. Canadian Journal of Biochemistry and Physiology, 37(8), 911-917. [PubMed: 13671378]

Brown AM, Evans RD, Black J, \& Ransom BR (2012). Schwann cell glycogen selectively supports myelinated axon function. Annals of Neurology, 72(3), 406-418. 10.1002/ana.23607 [PubMed: 23034913]

Brushart TM, Aspalter M, Griffin JW, Redett R, Hameed H, Zhou C, ... Hoke A (2013). Schwann cell phenotype is regulated by axon modality and central-peripheral location, and persists in vitro. Experimental Neurology, 247, 272-281. 10.1016/j.expneurol.2013.05.007 [PubMed: 23707299]

Capodivento G, Visigalli D, Garnero M, Fancellu R, Ferrara MD, Basit A, ... Nobbio L (2017). Sphingomyelin as a myelin biomarker in CSF of acquired demyelinating neuropathies. Scientific Reports, 7(1), 7831 10.1038/s41598-017-08314-1 [PubMed: 28798317]

Carrasco S, \& Merida I (2007). Diacylglycerol, when simplicity becomes complex. Trends in Biochemical Sciences, 32(1), 27-36. 10.1016/j.tibs.2006.11.004 [PubMed: 17157506]

Chen ZL, Yu WM, \& Strickland S (2007). Peripheral regeneration. Annual Review of Neuroscience, 30, 209-233. 10.1146/annurev.neuro.30.051606.094337

Cheng L, \& Mudge AW (1996). Cultured Schwann cells constitutively express the myelin protein P0. Neuron, 16(2), 309-319. [PubMed: 8789946]

Chiorazzi A, Wozniak KM, Rais R, Wu Y, Gadiano AJ, Farah MH, ... Cavaletti G (2018). Ghrelin agonist HM01 attenuates chemotherapy-induced neurotoxicity in rodent models. European Journal of Pharmacology, 840, 89-103. 10.1016/j.ejphar.2018.09.029 [PubMed: 30268665]

Cragnolini AB, \& Friedman WJ (2008). The function of p75NTR in glia. Trends in Neurosciences, 31(2), 99-104. 10.1016/j.tins.2007.11.005 [PubMed: 18199491]

Di Iorio A, Cherubini A, Volpato S, Sparvieri E, Lauretani F, Franceschi C, ... Ferrucci L (2006). Markers of inflammation, vitamin $\mathrm{E}$ and peripheral nervous system function: The InCHIANTI 
study. Neurobiology of Aging, 27(9), 1280-1288. 10.1016/j.neurobiolaging.2005.07.004 [PubMed: 16112778]

Domenech-Estevez E, Baloui H, Repond C, Rosafio K, Medard JJ, Tricaud N, ... Chrast R (2015). Distribution of monocarboxylate transporters in the peripheral nervous system suggests putative roles in lactate shuttling and myelination. The Journal of Neuroscience, 35 (10), 4151-4156. 10.1523/JNEUROSCI.3534-14.2015 [PubMed: 25762662]

Feltri ML, D’Antonio M, Previtali S, Fasolini M, Messing A, \& Wrabetz L (1999). P0-Cre transgenic mice for inactivation of adhesion molecules in Schwann cells. Annals of the New York Academy of Sciences, 883(1), 116-123. 10.1111/j.1749-6632.1999.tb08574.x

Fields RD (2015). A new mechanism of nervous system plasticity: Activity-dependent myelination. Nature Reviews. Neuroscience, 16(12), 756-767. 10.1038/nrn4023 [PubMed: 26585800]

Froehner SC, Davies A, Baldwin SA, \& Lienhard GE (1988). The blood-nerve barrier is rich in glucose transporter. Journal of Neurocytology, 17(2), 173-178. [PubMed: 3060567]

Funfschilling U, Supplie LM, Mahad D, Boretius S, Saab AS, Edgar J, ... Nave KA (2012). Glycolytic oligodendrocytes maintain myelin and long-term axonal integrity. Nature, 485(7399), 517-521. 10.1038/nature11007 [PubMed: 22622581]

Garbay B, Heape AM, Sargueil F, \& Cassagne C (2000). Myelin synthesis in the peripheral nervous system. Progress in Neurobiology, 61(3), 267-304. [PubMed: 10727776]

Georgiou J, \& Charlton MP (1999). Non-myelin-forming perisynaptic Schwann cells express protein zero and myelin-associated glycoprotein. Glia, 27(2), 101-109. [PubMed: 10417810]

Gerhart DZ, \& Drewes LR (1990). Glucose transporters at the blood-nerve barrier are associated with perineurial cells and endoneurial microvessels. Brain Research, 508(1), 46-50. [PubMed: 2337790]

Hoke A, Redett R, Hameed H, Jari R, Zhou C, Li ZB, ... Brushart TM (2006). Schwann cells express motor and sensory phenotypes that regulate axon regeneration. The Journal of Neuroscience, 26(38), 9646-9655. 10.1523/JNEUROSCI.1620-06.2006 [PubMed: 16988035]

Ichihara Y, Doi T, Ryu Y, Nagao M, Sawada Y, \& Ogata T (2017). Oligodendrocyte progenitor cells directly utilize lactate for promoting cell cycling and differentiation. Journal of Cellular Physiology, 232(5), 986-995. 10.1002/jcp.25690 [PubMed: 27861886]

Jessen KR, \& Mirsky R (2008). Negative regulation of myelination: Relevance for development, injury, and demyelinating disease. Glia, 56(14), 1552-1565. 10.1002/glia.20761 [PubMed: 18803323]

Jha MK, \& Morrison BM (2018). Glia-neuron energy metabolism in health and diseases: New insights into the role of nervous system metabolic transporters. Experimental Neurology, 309, 23-31. 10.1016/j.expneurol.2018.07.009 [PubMed: 30044944]

Jolliffe I (2011). Principal component analysis In International encyclopedia of statistical science (pp. 1094-1096). New York: Springer.

Katona I, \& Weis J (2017). Diseases of the peripheral nerves. Handbook of Clinical Neurology, 145, 453-474. 10.1016/B978-0-12-802395-2.00031-6 [PubMed: 28987189]

Kihara M, Nickander KK, \& Low PA (1991). The effect of aging on endoneurial blood flow, hyperemic response and oxygen-free radicals in rat sciatic nerve. Brain Research, 562(1), 1-5. [PubMed: 1799863]

Lee Y, Morrison BM, Li Y, Lengacher S, Farah MH, Hoffman PN, ... Rothstein JD (2012). Oligodendroglia metabolically support axons and contribute to neurodegeneration. Nature, 487(7408), 443-448. 10.1038/nature11314 [PubMed: 22801498]

Leite TC, Coelho RG, Da Silva D, Coelho WS, Marinho-Carvalho MM, \& Sola-Penna M (2011). Lactate downregulates the glycolytic enzymes hexokinase and phosphofructokinase in diverse tissues from mice. FEBS Letters, 585(1), 92-98. 10.1016/j.febslet.2010.11.009 [PubMed: 21074528]

Low PA, Schmelzer JD, \& Ward KK (1986). The effect of age on energy metabolism and resistance to ischaemic conduction failure in rat peripheral nerve. The Journal of Physiology, 374, 263-271. [PubMed: 3746689] 
Magnani P, Cherian PV, Gould GW, Greene DA, Sima AA, \& Brosius FC 3rd. (1996). Glucose transporters in rat peripheral nerve: Paranodal expression of GLUT1 and GLUT3. Metabolism, 45(12), 1466-1473. [PubMed: 8969278]

MathWorks. (2018). MATLAB and statistics toolbox release 2018b [program. Natick, MA: The MathWorks.

Montani L, Pereira JA, Norrmen C, Pohl HBF, Tinelli E, Trotzmuller M, ... Suter U (2018). De novo fatty acid synthesis by Schwann cells is essential for peripheral nervous system myelination. The Journal of Cell Biology, 217(4), 1353-1368. 10.083/jcb.201706010 [PubMed: 29434029]

Morrison BM, Tsingalia A, Vidensky S, Lee Y, Jin L, Farah MH, ... Rothstein JD (2015). Deficiency in monocarboxylate transporter 1 (MCT1) in mice delays regeneration of peripheral nerves following sciatic nerve crush. Experimental Neurology, 263, 325-338. 10.1016/ j.expneurol.2014.10.018 [PubMed: 25447940]

Nguyen T, Mehta NR, Conant K, Kim KJ, Jones M, Calabresi PA, ... Griffin JW (2009). Axonal protective effects of the myelin-associated glycoprotein. The Journal of Neuroscience, 29(3), 630 637. 10.1523/JNEUROSCI.5204-08.2009 [PubMed: 19158290]

O’Brien JS, Sampson EL, \& Stern MB (1967). Lipid composition of myelin from the peripheral nervous system. Intradural spinal roots. Journal of Neurochemistry, 14(3), 357-365. [PubMed: 6020462]

Painter MW, Brosius Lutz A, Cheng YC, Latremoliere A, Duong K, Miller CM, ... Woolf CJ (2014). Diminished Schwann cell repair responses underlie age-associated impaired axonal regeneration. Neuron, 83(2), 331-343. 10.1016/j.neuron.2014.06.016 [PubMed: 25033179]

Patti GJ, Yanes O, Shriver LP, Courade JP, Tautenhahn R, Manchester M, \& Siuzdak G (2012). Metabolomics implicates altered sphingolipids in chronic pain of neuropathic origin. Nature Chemical Biology, 8(3), 232-234. 10.1038/nchembio.767 [PubMed: 22267119]

Pellerin L, \& Magistretti PJ (1994). Glutamate uptake into astrocytes stimulates aerobic glycolysis: A mechanism coupling neuronal activity to glucose utilization. Proceedings of the National Academy of Sciences of the United States of America, 91(22), 10625-10629. [PubMed: 7938003]

Qureshi MA, Haynes CM, \& Pellegrino MW (2017). The mitochondrial unfolded protein response: Signaling from the powerhouse. The Journal of Biological Chemistry, 292(33), 13500-13506. 10.1074/jbc.R117.791061 [PubMed: 28687630]

Rice JA (2007). Mathematical statistics and data analysis (3rd ed.). Belmont, CA: Thomson/Brooks/ Cole.

Riviere L, Moreau P, Allmann S, Hahn M, Biran M, Plazolles N, ... Bringaud F (2009). Acetate produced in the mitochondrion is the essential precursor for lipid biosynthesis in procyclic trypanosomes. Proceedings of the National Academy of Sciences of the United States of America, 106(31), 12694-12699. 10.1073/pnas.0903355106 [PubMed: 19625628]

Rohart F, Gautier B, Singh A, \& Le Cao KA (2017). mixOmics: An R package for 'omics feature selection and multiple data integration. PLoS Computational Biology, 13(11), e1005752 10.1371/ journal.pcbi.1005752 [PubMed: 29099853]

Sasaki Y, Hackett AR, Kim S, Strickland A, \& Milbrandt J (2018). Dysregulation of NAD(+) metabolism induces a Schwann cell dedifferentiation program. The Journal of Neuroscience, 38(29), 6546-6562. 10.1523/JNEUROSCI.3304-17.2018 [PubMed: 29921717]

Scheib JL, \& Hoke A (2016). An attenuated immune response by Schwann cells and macrophages inhibits nerve regeneration in aged rats. Neurobiology of Aging, 45, 1-9. 10.1016/ j.neurobiolaging.2016.05.004 [PubMed: 27459920]

Schmitt S, Castelvetri LC, \& Simons M (2015). Metabolism and functions of lipids in myelin. Biochimica et Biophysica Acta, 1851(8), 999-1005. 10.1016/j.bbalip.2014.12.016 [PubMed: 25542507]

Skarnes WC, Rosen B, West AP, Koutsourakis M, Bushell W, Iyer V, ... Bradley A (2011). A conditional knockout resource for the genome-wide study of mouse gene function. Nature, 474(7351), 337-342. 10.1038/nature10163 [PubMed: 21677750]

Stabler SP (2013). Clinical practice. Vitamin B12 deficiency. The New England Journal of Medicine, 368(2), 149-160. 10.1056/NEJMcp1113996 [PubMed: 23301732] 
Stockstill K, Doyle TM, Yan X, Chen Z, Janes K, Little JW, ... Salvemini D (2018). Dysregulation of sphingolipid metabolism contributes to bortezomib-induced neuropathic pain. The Journal of Experimental Medicine, 215(5), 1301-1313. 10.1084/jem.20170584 [PubMed: 29703731]

Takebe K, Nio-Kobayashi J, Takahashi-Iwanaga H, \& Iwanaga T (2008). Histochemical demonstration of a monocarboxylate transporter in the mouse perineurium with special reference to GLUT1. Biomedical Research, 29(6), 297-306. [PubMed: 19129673]

Turner DA, \& Vevallos WH (1968). Vitamin B12 and the lipids of the central nervous system. Clinical Biochemistry, 2, 1-11.

Vega C, Martiel JL, Drouhault D, Burckhart MF, \& Coles JA (2003). Uptake of locally applied deoxyglucose, glucose and lactate by axons and Schwann cells of rat vagus nerve. The Journal of Physiology, 546(Pt. 2), 551-564. [PubMed: 12527741]

Verdin E (2015). NAD(+) in aging, metabolism, and neurodegeneration. Science, 350(6265), 12081213. 10.1126/science.aac4854 [PubMed: 26785480]

Viader A, Golden JP, Baloh RH, Schmidt RE, Hunter DA, \& Milbrandt J (2011). Schwann cell mitochondrial metabolism supports long-term axonal survival and peripheral nerve function. The Journal of Neuroscience, 31(28), 10128-10140. 10.1523/JNEUROSCI.0884-11.2011 [PubMed: 21752989]

Viader A, Sasaki Y, Kim S, Strickland A, Workman CS, Yang K, ... Milbrandt J (2013). Aberrant Schwann cell lipid metabolism linked to mitochondrial deficits leads to axon degeneration and neuropathy. Neuron, 77(5), 886-898. 10.1016/j.neuron.2013.01.012 [PubMed: 23473319]

Walsh ME, Sloane LB, Fischer KE, Austad SN, Richardson A, \& Van Remmen H (2015). Use of nerve conduction velocity to assess peripheral nerve health in aging mice. The Journals of Gerontology. Series A, Biological Sciences and Medical Sciences, 70(11), 1312-1319. 10.1093/gerona/glu208

Wang G, Cao L, Wang Y, Hua Y, Cai Z, Chen J, ... Shen Z (2017). Human eyelid adipose tissuederived Schwann cells promote regeneration of a transected sciatic nerve. Scientific Reports, 7, 43248 10.1038/srep43248 [PubMed: 28256528]

Weyer AD, Zappia KJ, Garrison SR, O’Hara CL, Dodge AK, \& Stucky CL (2016). Nociceptor sensitization depends on age and pain chronicity(1,2,3). eNeuro, 3(1), 1-26. 10.1523/ ENEURO.0115-15.2015

Wright MC, Mi R, Connor E, Reed N, Vyas A, Alspalter M, ... Hoke A (2014). Novel roles for osteopontin and clusterin in peripheral motor and sensory axon regeneration. The Journal of Neuroscience, 34(5), 1689-1700. 10.1523/JNEUROSCI.3822-13.2014 [PubMed: 24478351]

Yang Y, Lane AN, Ricketts CJ, Sourbier C, Wei MH, Shuch B, ... Linehan WM (2013). Metabolic reprogramming for producing energy and reducing power in fumarate hydratase null cells from hereditary leiomyomatosis renal cell carcinoma. PLoS One, 8(8), e72179 10.1371/ journal.pone.0072179 [PubMed: 23967283]

Yin X, Kidd GJ, Ohno N, Perkins GA, Ellisman MH, Bastian C, ... Trapp BD (2016). Proteolipid protein-deficient myelin promotes axonal mitochondrial dysfunction via altered metabolic coupling. The Journal of Cell Biology, 215(4), 531-542. 10.1083/jcb.201607099 [PubMed: 27872255] 
(a)

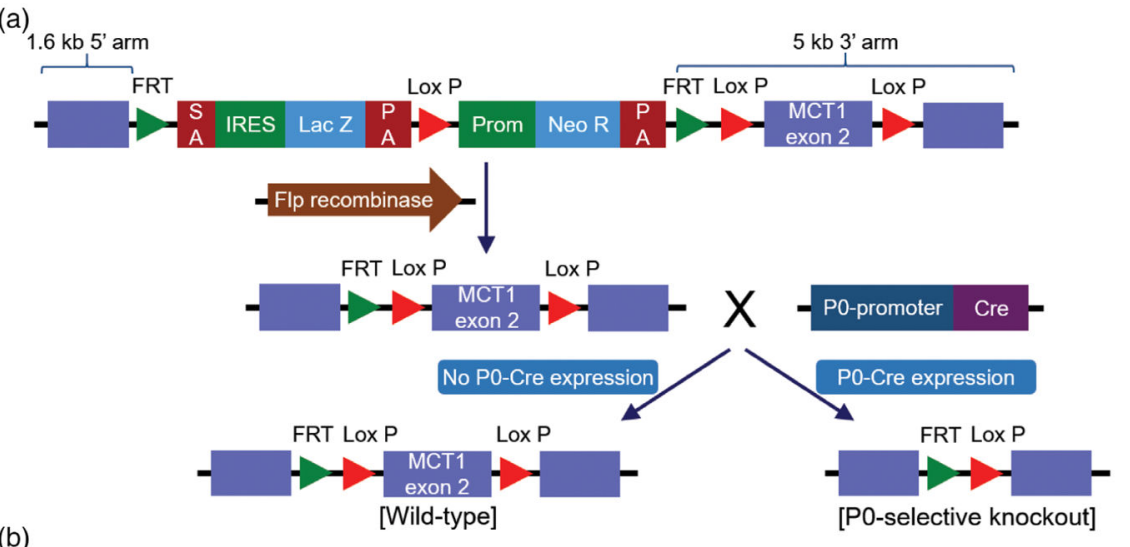

(b)
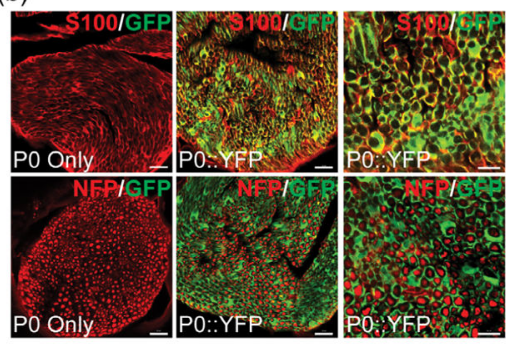

(c) Wild-type $\mathrm{P} 0-\mathrm{CMCT} 1 \mathrm{KO}$

(d)
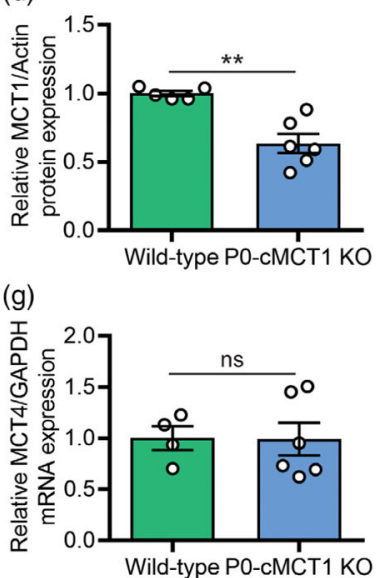

(e)
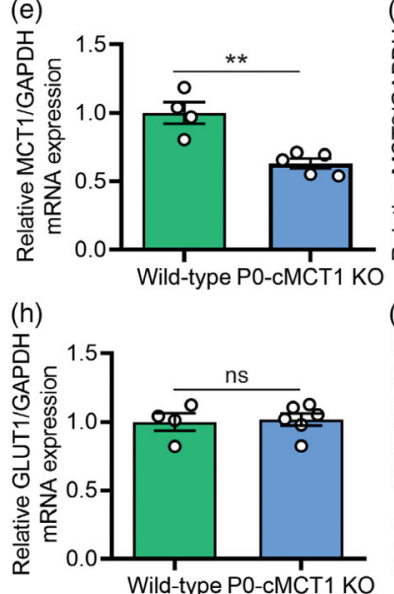
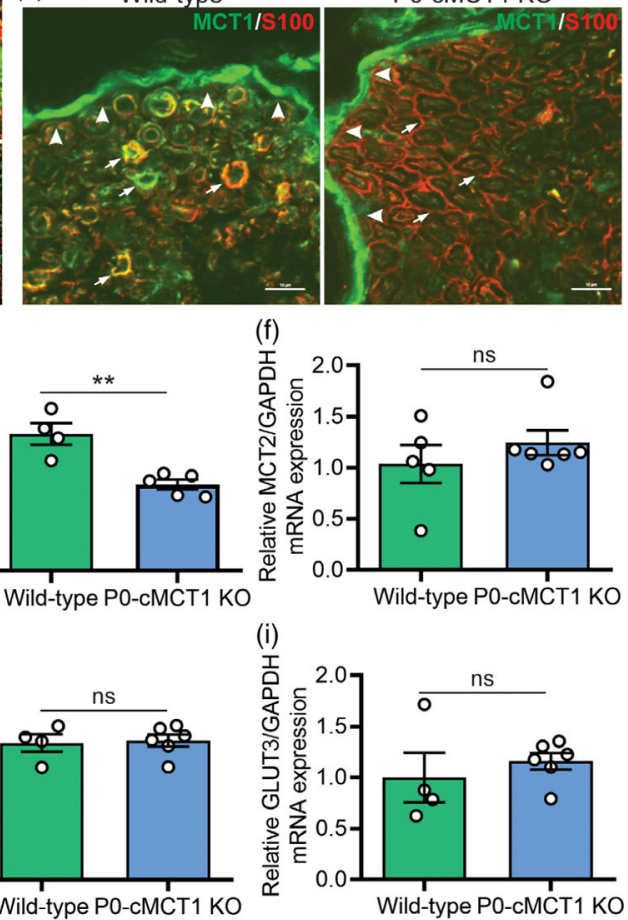

FIGURE 1.

Production and validation of P0-cMCT1 KO mice. (a) P0-cMCT1 KO (P0Cre:MCT1 ${ }^{f l / f 1}$ ) mice were generated by crossing our laboratory-developed $\mathrm{MCT} 1^{\mathrm{f} / \mathrm{f}}$ mice having MCT1 exon2 inserted in between two LoxP sites with P0-Cre transgenic mice. (b) $\mathrm{P} 0-\mathrm{Cre}^{+/-}$, RosaYFP mice show complete co-localization of P0-Cre expression (GFP) with S100 (red), a marker for SCs. There is no co-localization of P0-Cre expression (GFP) with phosphorylated neurofilament (NFP; red), which labels axons. Scale bars, $20 \mu \mathrm{m}$ for left and mid panels; $10 \mu \mathrm{m}$ for right panel. (c) MCT1 immunoreactivity (IR; green) in perineurial cells (arrowheads) and SCs (arrows), co-localized with S100 (red) in wild-type nerves (P0:MCT1 $1^{w t / w t}$ or wild-type). MCT1 IR (green) is absent from SCs labeled with S100 (red; arrows), but not perineurial cells (arrowheads), in P0-cMCT1 KO mice nerves. Scale bar, 10 $\mu \mathrm{m}$. (d-i) SC specific MCT1 deletion downregulates the expression of MCT1 protein (d) and mRNA (e) without altering the expression of MCT2 (f), MCT4 (g), GLUT1 (h), or GLUT3 
(i) in peripheral nerves of P0-cMCT1 KO mice. Levels of mRNAs are depicted as fold change compared with wild-type mice normalized to their corresponding GAPDH mRNA levels. Mean $\pm S E M, n=4-6$ per group, ${ }^{* *} p<.01 ; \mathrm{ns}=$ not significant, unpaired $t$ test 
(a)

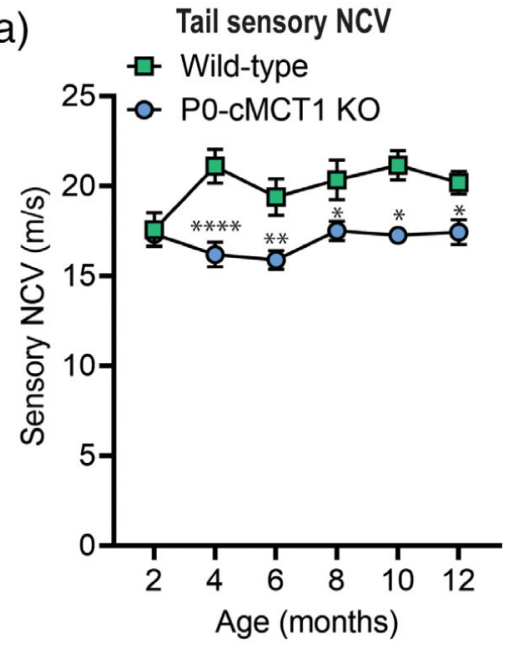

(b) Tail sensory evoked potential amplitude

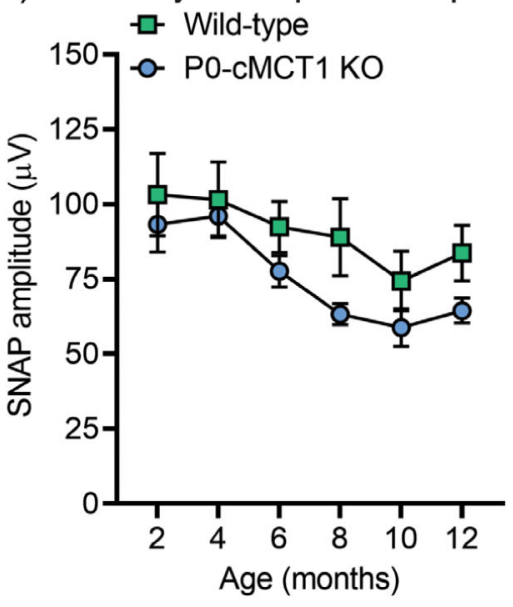

(c)

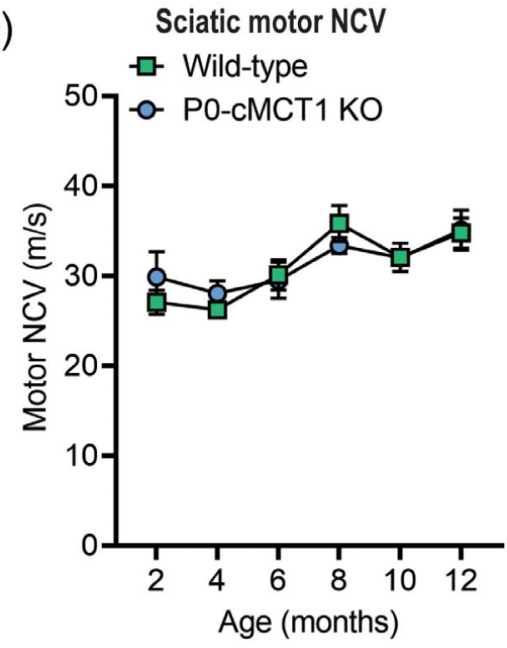

(d) Sciatic motor evoked potential amplitude

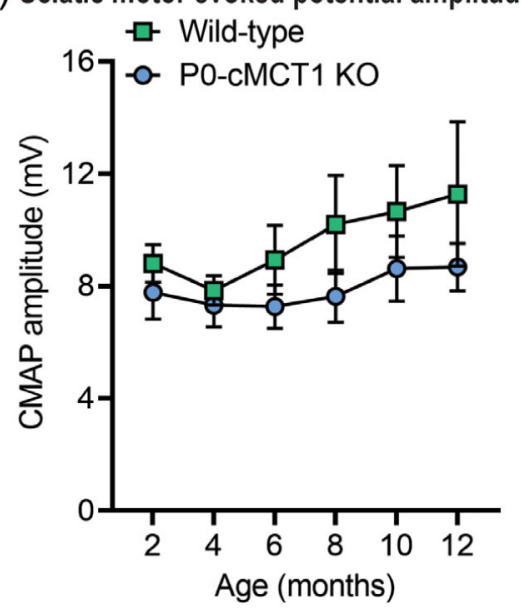

FIGURE 2.

Sensory, but not motor, NCV is decreased in maturing P0-cMCT1 KO mice. (a and c) SCspecific MCT1 deletion reduces the sensory (a), but not motor (c), NCV in P0-cMCT1 KO mice, compared to wild-type littermate control mice, by 4 months of age. Mean $\pm S E M, n=$ 5-7 per group, ${ }^{*} p<.05, * * p<.01, * * * * p<.0001$; two-way ANOVA with Holm-Sidak's multiple comparisons test. (b and d) No significant impact of SC MCT1 deletion is observed on SNAP (b) and CMAP (d) amplitudes. The electrophysiologic recording was performed at room temperature, where tail temperature was usually recorded to be in between 22 and $24^{\circ} \mathrm{C}$. Mean $\pm S E M, n=5-7$ per group. CMAP, compound muscle action potential; NCV, nerve conduction velocity; SNAP, sensory nerve action potential 

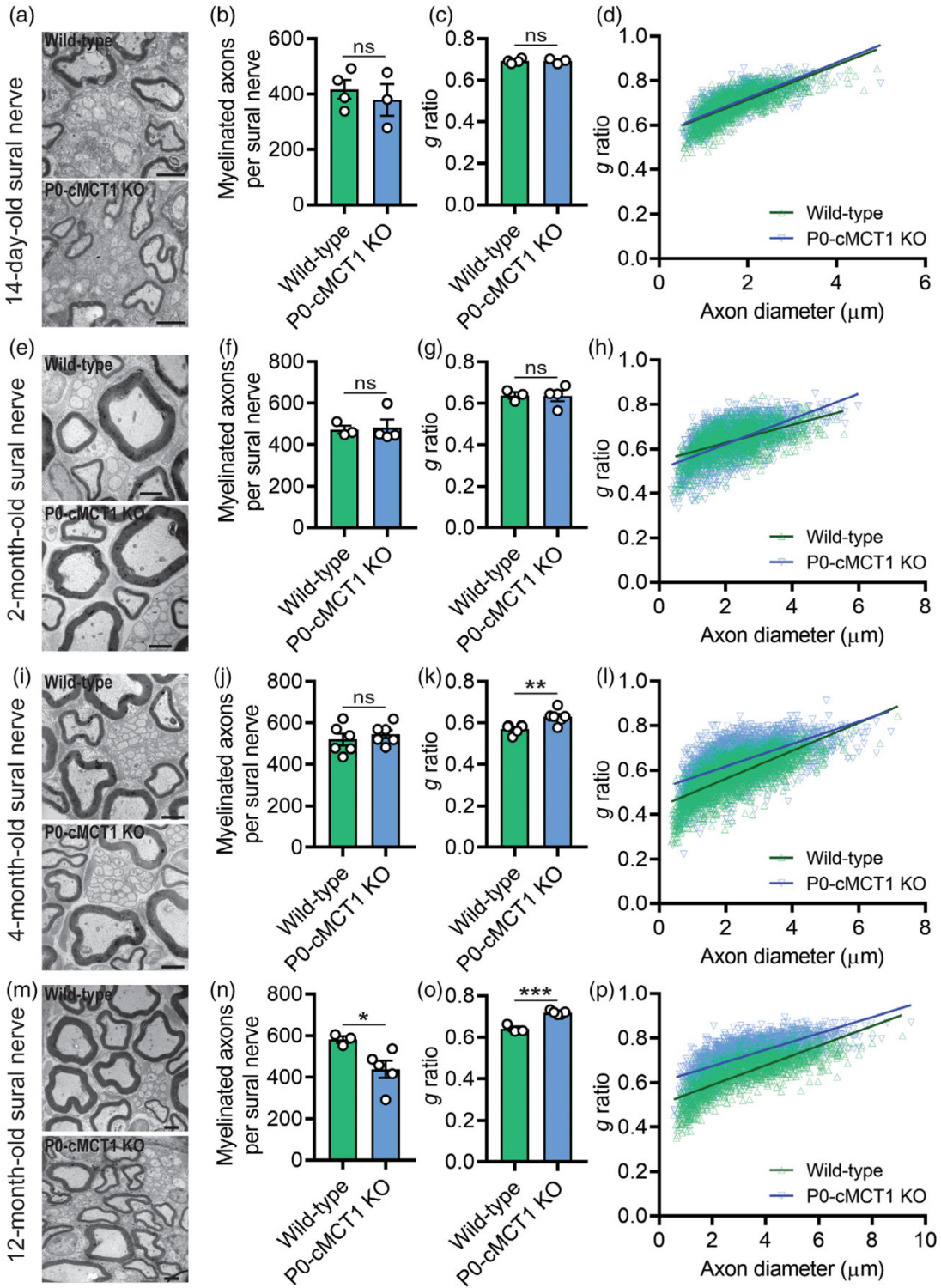

FIGURE 3.

$\mathrm{SC}$-specific MCT1 contributes to maintenance of sensory axon myelination. In 14-day-old (a-d) and 2-month-old (e-h) wild-type and P0-cMCT1 KO mice sural nerves, electron micrographs (a and e), myelinated axon count (b and f), $g$ ratio (c and g), and scatter plot graph displaying $g$ ratio ( $y$-axis) in relation to axon diameter ( $x$-axis) of individual fiber $(\mathrm{d}$ and $\mathrm{h}$ ) confirmed normal embryonic and early post-natal sural nerve development in P0cMCT1 KO mice. In 4-month-old wild-type and P0-cMCT1 KO mice sural nerves, electron micrographs (i), myelinated axon count (j), $g$ ratio (k), and scatter plot graph displaying $g$ ratio ( $y$-axis) in relation to axon diameter ( $x$-axis) of individual fiber (l) confirmed sural nerve hypomyelination in P0-cMCT1 KO mice. In 12-month-old wild-type and P0-cMCT1 KO mice sural nerves, electron micrographs (m), myelinated axon count (n), $g$ ratio (o), and scatter plot graph displaying $g$ ratio ( $y$-axis) in relation to axon diameter ( $x$-axis) of individual fiber (p) confirmed sural nerve hypomyelination in P0-cMCT1 KO mice. There 
was also a significant reduction in myelinated axons at 12 months (n), but not earlier time points, in P0-cMCT1 KO mice. Mean $\pm S E M, n=3-6$ per group, ${ }^{*} p<.05 ; * * p<.01 ; * * * p$ $<.001 ; \mathrm{ns}=$ not significant, unpaired $t$ test. Scale bar, $2 \mu \mathrm{m}$. The $g$ ratio between wild-type (green line) and P0-cMCT1 KO (blue line) was not different in 14-day-old (c) and 2-monthold mice (g) but was significantly different in 4-month-old (k) and 12-month-old (o) mice (for 14-day-old mice: $p>.05 ; t=0.6309$, for 2-month-old mice: $p>.05 ; t=3.010$, for 4 month-old mice: $p<.0001 ; t=29.29$, for 12-month-old mice: $p<.0001 ; t=30.30, d f=$ 16,066 , one-way ANOVA with Bonferroni's multiple comparisons test) 
(a)

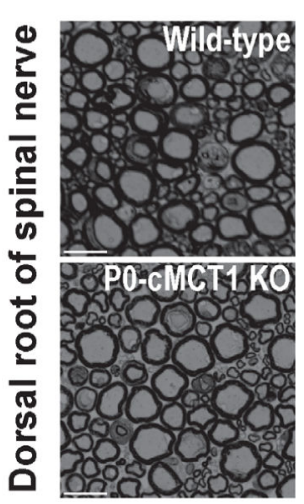

(e)

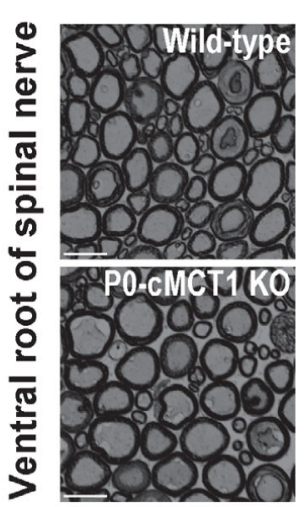

(b) $₫$

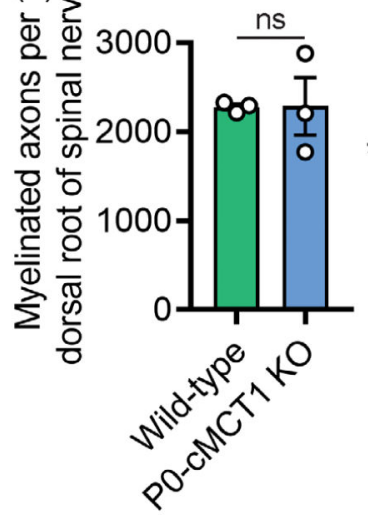

(f)

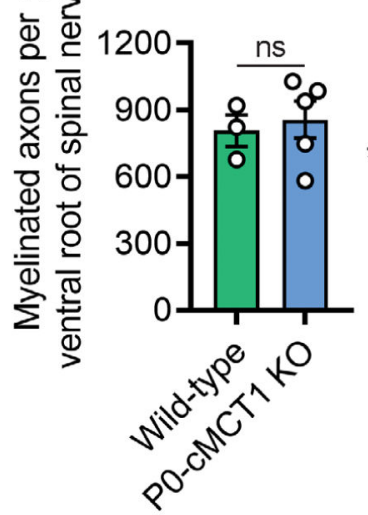

(c)

(d)
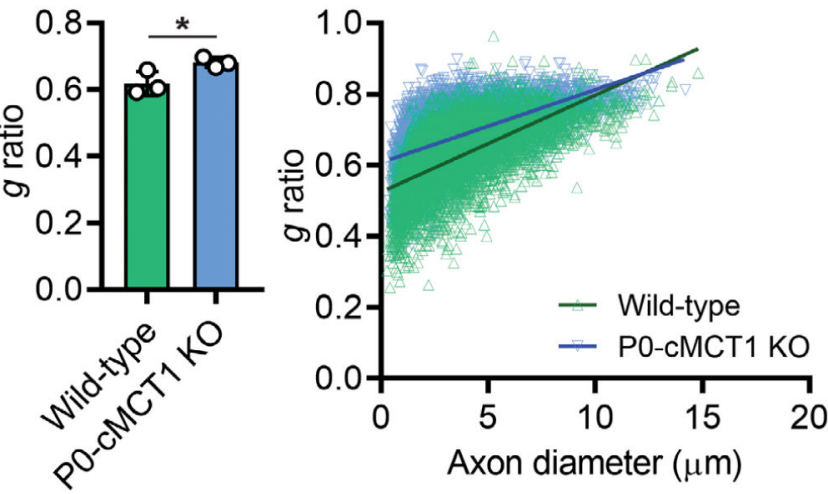

(g)

(h)

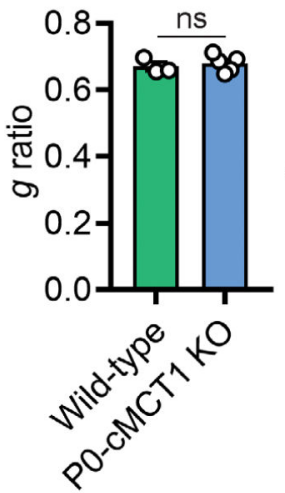

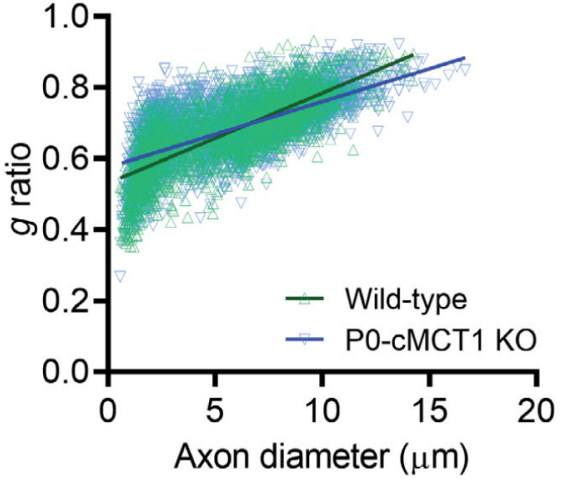

FIGURE 4.

SC-specific MCT1 is critical for myelin maintenance of dorsal, but not ventral, roots of spinal nerves. Photomicrographs (a), myelinated axon count (b), $g$ ratio (c), and scatter plot graph displaying $g$ ratio ( $y$-axis) in relation to axon diameter ( $x$-axis) of individual fiber $(d)$ demonstrated hypomyelination of dorsal spinal nerve roots, without myelinated axon loss, in 12-month old P0-cMCT1 KO mice. Photomicrographs (e), myelinated axon count (f), $g$ ratio $(\mathrm{g})$, and scatter plot graph displaying $g$ ratio ( $y$-axis) in relation to axon diameter ( $x$-axis) of individual fiber (h) confirmed no notable difference between ventral spinal nerve roots of 12-month-old P0-cMCT1 KO mice when compared to wild-type mice. Light microscope photomicrographs and subsequent analysis completed on toluidine blue-stained sections. Mean $\pm S E M, n=3-5$ per group, ${ }^{*} p<.05 ; * * p<.01 ; * * *<.001 ; \mathrm{ns}=$ not significant, unpaired $t$ test. Scale bar, $20 \mu \mathrm{m}$ 

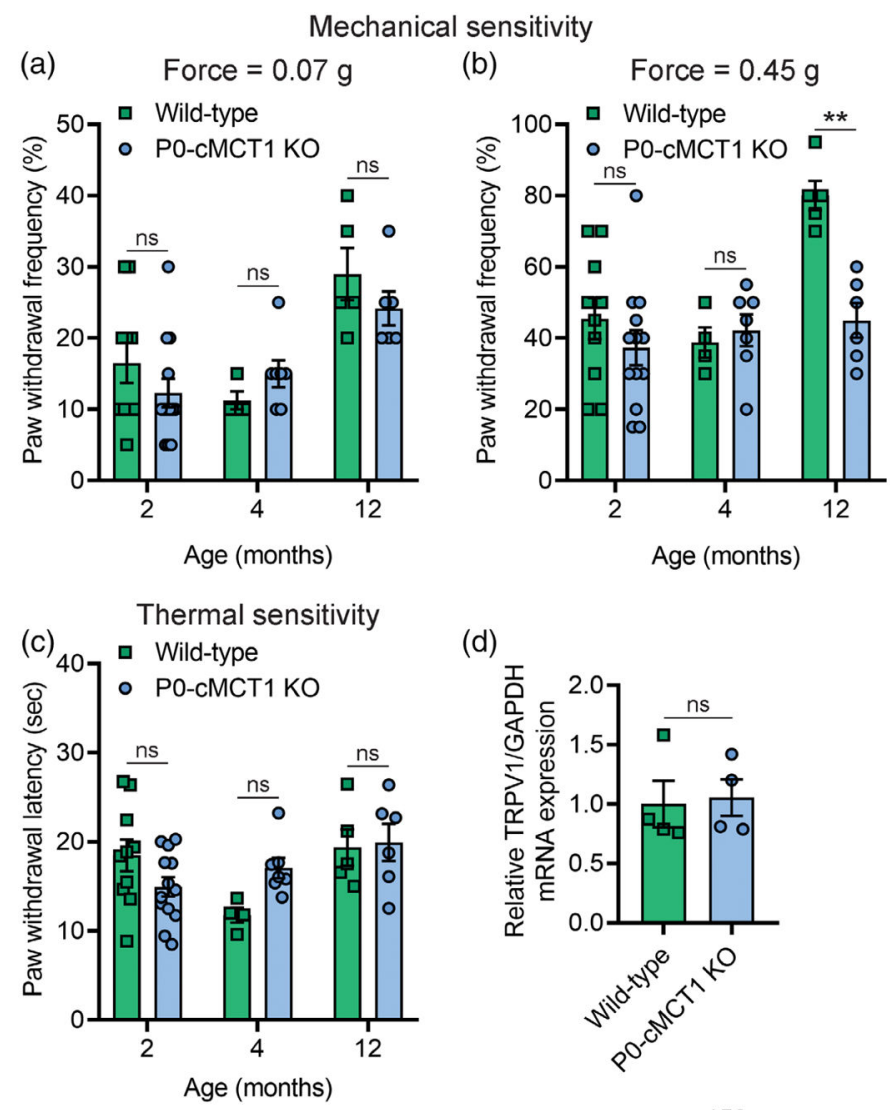

(d)
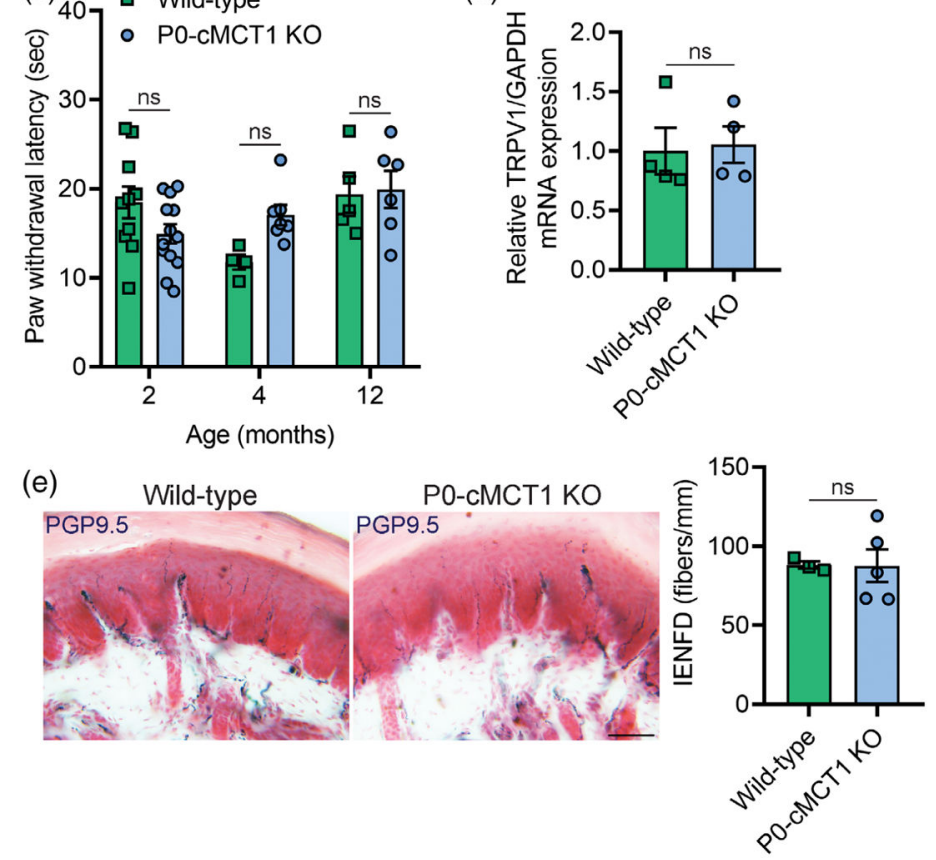

FIGURE 5.

Mechanical sensitivity is impaired in SC-specific MCT1 deficient mice. Assessment of paw withdrawal frequency to mechanical stimulation by calibrated von Frey monofilaments of forces $0.07 \mathrm{~g}$ (a) and $0.45 \mathrm{~g}$ (b) in wild-type and P0-cMCT1 KO mice. (c) Assessment of thermal sensitivity by using radiant paw-heating assay. Current set at baseline level: $20 \%$, 10-12 s; cut off time; $30 \mathrm{~s}$. Mean $\pm S E M, n=4-10$ for wild-type and 6-13 for P0-cMCT1 KO groups, ${ }^{* *} p<.01 ; \mathrm{ns}=$ not significant, two-way ANOVA with Bonferroni's multiple comparisons test. (d) Assessment of the TRPV1 mRNA expression by qPCR in sciatic nerves of 4-month-old wild-type and P0-cMCT1 KO mice. Levels of TRPV1 mRNA are depicted as fold change compared with wild-type mice normalized to its corresponding GAPDH mRNA levels. $n=4$ per group, $\mathrm{ns}=$ not significant, unpaired $t$ test, mean $\pm S E M$. (e) Images (left panel) showing representative picture of skin biopsies and quantification of IENFD (right panel) obtained from the footpads of 4-month-old wild-type and P0-cMCT1 
KO mice following immunohistochemical staining for PGP9.5. $n=3$ for wild-type and 5 for P0-cMCT1 KO groups, ns = not significant, unpaired $t$ test, mean $\pm S E M$. Scale bar, $50 \mu \mathrm{m}$ 

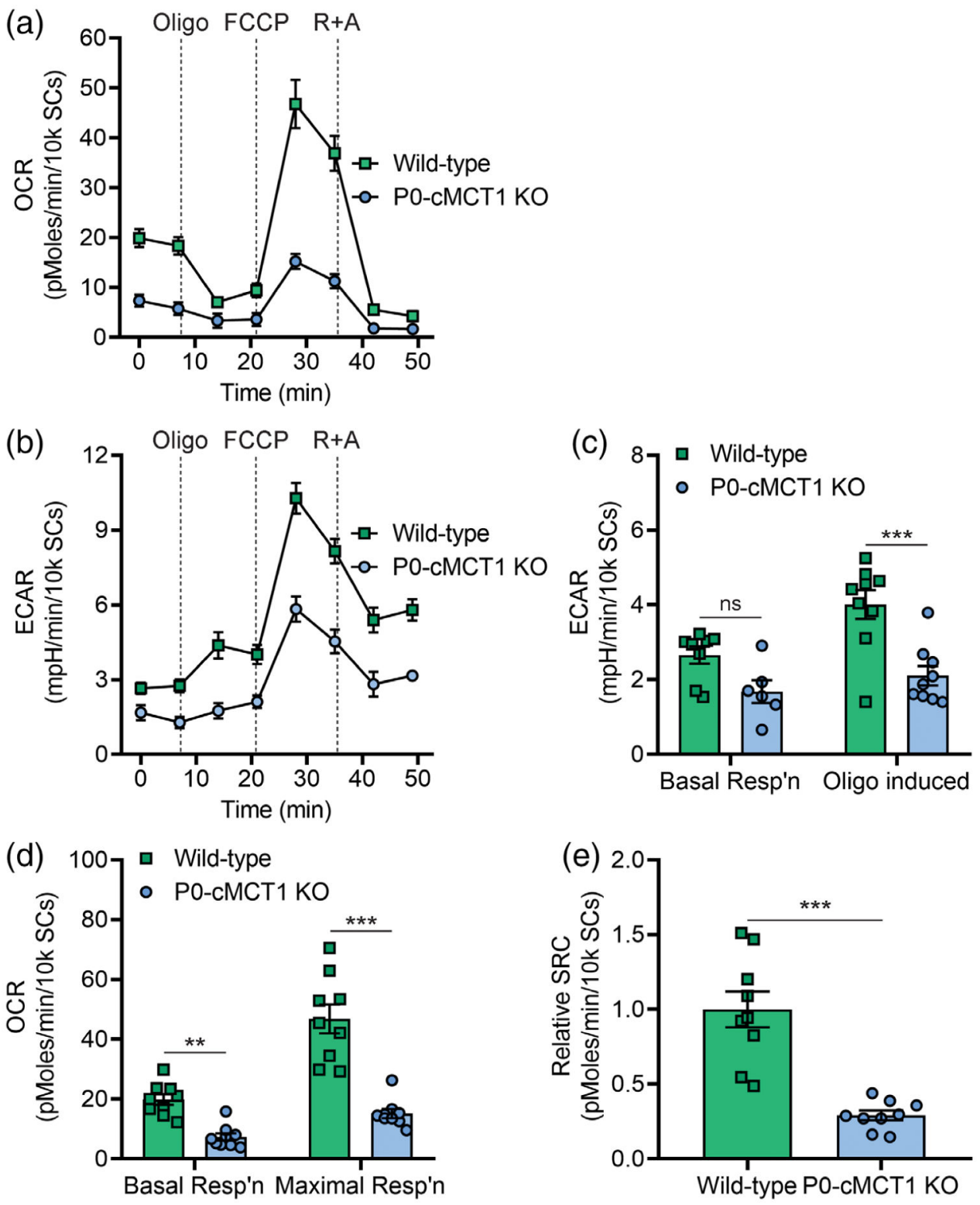

FIGURE 6.

Selective downregulation of MCT1 within SCs impairs glycolytic and mitochondrial functions. Real-time oxygen consumption (a) and extracellular acidification (b) were measured in SCs isolated from sciatic nerves of 4-week-old wild-type and P0-cMCT1 KO littermate mice with the Seahorse extracellular flux analyzer. The ECAR was compared between wild-type and P0-cMCT1 KO SCs during basal condition and following oligomycin treatment (c). The OCR was compared between wild-type and P0-cMCT1 KO SCs during basal respiration and FCCP-induced maximal respiration (d). Spare respiratory capacity (SRC) was calculated as the difference between maximal respiration and basal respiration (e). Mean $\pm S E M, n=9$ per group, ${ }^{* *} p<.01 ; * * * p<.001$; ns = not significant, two-way ANOVA with Bonferroni's multiple comparisons test for (c) and (d); unpaired $t$ test for (e). ECAR, extracellular acidification rate; FCCP, carbonyl cyanide-4 (trifluoromethoxy) 
phenylhydrazone; OCR, oxygen consumption rate; Oligo, oligomycin; R+A; Rotenone and Antimycin A 
(a)

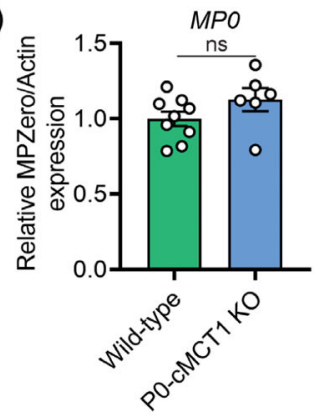

(d)

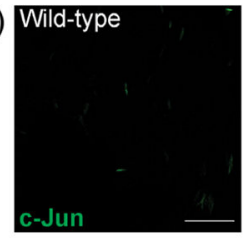

(e)

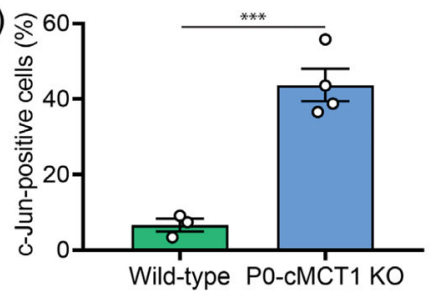

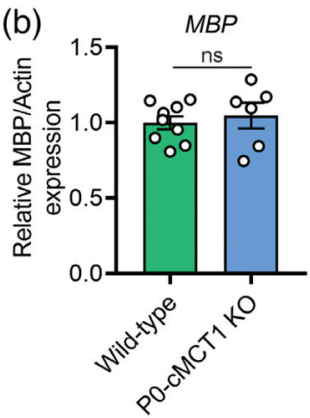

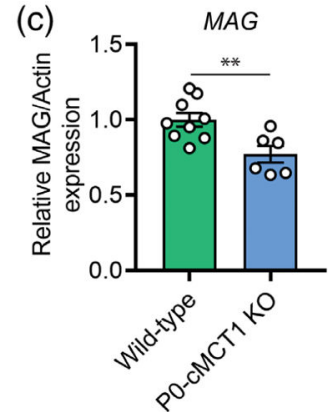

(g)

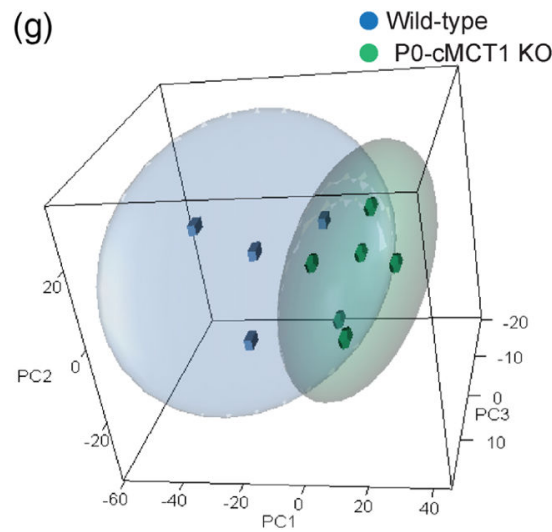

(f)

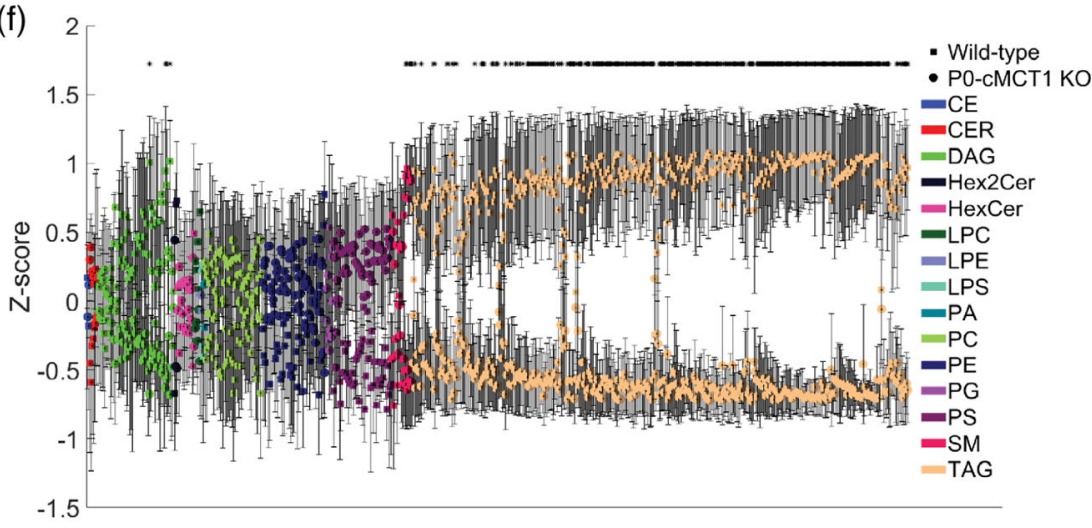

FIGURE 7.

SC-specific MCT1 deficiency decreases the synthesis of myelin-associated glycoprotein (MAG) as well as lipogenesis in peripheral nerves during aging. Expression of myelin protein zero ( $\mathrm{P} 0, \mathrm{a})$, myelin basic protein (MBP, b), and MAG (c) in sciatic nerve homogenates normalized to actin are presented as fold change relative to wild-type control. The full-length Western blots used for the densitometry quantification are shown in Figure S7. Mean $\pm S E M, \mathrm{n}=9$ for wild-type and 6 for P0-cMCT1 KO groups, ${ }^{* *} p<.01$; ns $=$ not significant, unpaired t test. (d) Representative images of longitudinal sections of sciatic nerves of mature (4-month-old) wild-type and P0-cMCT1 KO mice immunostained for cJun (green). (e) Quantification of the c-Jun positive cells in the immunostained longitudinal sections of sciatic nerves presented in panel d. c-Jun-positive cells are presented as a percentage relative to the number of DAPI-stained nuclei. Mean $\pm S E M, n=3$ for wild-type and 4 for P0-cMCT1 KO groups, ${ }^{* * *} p<.001$, unpaired $t$ test. (f and $\mathrm{g}$ ) Lipidomics analysis 
of sural nerves isolated from 8-month old wild-type and P0-cMCT1 KO mice. (f) Z-score plot of group means for all detected lipid species. Lipid classes are identified by the indicated color with wild-type $=$ circles, and P0-cMCT1 KO $=$ squares. Please see Supporting Information Table S1 for clear identification of individual lipids. Mean $\pm S E M$ ${ }^{*} p<.05, \mathrm{t}$ test with Benjamini-Hochberg correction for multiple comparisons. (g) Scatter plot of primary component analyses (PCA) scores for wild-type blue cubes and P0-cMCT1 KO green spheres groups. Ellipses represent $95 \%$ confidence intervals. $n=4$ for wild-type and 6 for P0-cMCT1 KO groups 


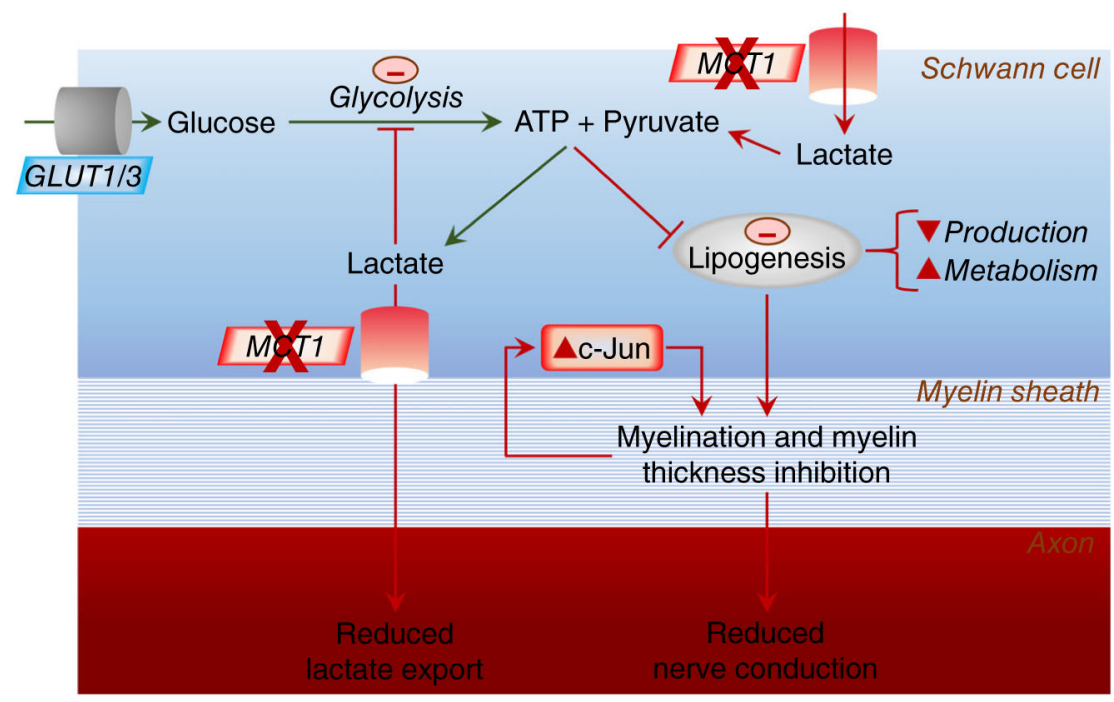

FIGURE 8.

Model for impact of SC ablation of MCT1 on lipogenesis, c-Jun, and axon-myelin function. Circulating glucose is taken up into SCs by glucose transporters, both GLUT1 and GLUT3. Glucose is metabolized to pyruvate and ATP is produced via glycolysis. Besides generating more ATP via oxidative phosphorylation in mitochondria, some of the pyruvate is converted to lactate. Lactate can also be directly imported into SCs from the circulation and extracellular space. This lactate can be converted to pyruvate for subsequent oxidative phosphorylation in SCs. In the absence of MCT1, the lactate transport into SCs is disrupted, which can impair lipogenesis, lead to elevated c-Jun, and result in immature, poorly myelinating SCs 